\title{
Multiplicity of Filtered Rings and Simple K3 Singularities of Multiplicity Two
}

\author{
By \\ Masataka TOMARI*
}

\begin{abstract}
Given a filtered ring, we give bounds of its multiplicity in terms of the data of the tangent cone using the technique of the filtered blowing-up. Applying it to each simple K3 singularity of multiplicity two, we find a good coordinate where the Newton boundary of the defining equation contains the point $(1,1,1,1) \in \mathbf{R}^{4}$. In the course of the proof, we classify simple K3 singularities of multiplicity two into 48 weight types. Furthermore we prove that the weight type of the singularity stays the same under arbitrary one-parameter (FG)-deformations.
\end{abstract}

\section{Contents}

Chapter I. Multiplicity of Filtered Rings

$\S 1$. A Proof of Theorem A

$\S 2$. Remarks on Normal Graded Rings

Chapter II. The Inequality $q_{1} q_{2} q_{3} D^{2} \geq 2$ for Normal K3 Surfaces

$\S 3 . \quad$ Some Easy Cases

$\S 4$. The Case $b_{1}=b_{2}=0$ and $b_{3} \leq 1, b_{4} \leq 1$

$\S 5$. The Case of $b_{1}=0, b_{2}=1$ and $b_{3} \leq 1$

$\S 6$. In the Case with $b_{1}=b_{2}=1$

$\S 7 . \quad$ Simple K3 Singularities of Multiplicity Two

Communicated by S. Mori. Received August 29, 2000.

2000 Mathematics Subject Classification(s): Primary 14B05; Secondary 14H20, 14J17.

Key words and phrase: Multiplicity, filtered rings, Simple K3 singularity.

*Department of Mathematics, Faculty of Sciences, Kanazawa University, Kakuma-Machi,

Kanazawa 920-1192, Japan.

e-mail: tomari@kenroku.kanazawa-u.ac.jp

fax: (Japan) 076-264-5738 
References

\section{Introduction}

The study of various blowing-ups is very important in the theory of singularities. In many cases some blowing-up appears as the blowing-down of divisors of algebraic variety, and is understood naturally as a filtered blowingup. As a continuation of [20] and [19], we will study the multiplicity of the singularity from the point of the theory of filtered rings. Let $(V, p)$ be a germ of a projective variety at a closed point $p$, and $(A, m)$ the local ring of $(V, p)$. We consider the filtration $F=\left\{F^{k}\right\}$ of ideals of $A$ which satisfies; $F^{0}=A$, $F^{1}=m, F^{k} \supset F^{k+1}, F^{k} \cdot F^{j} \subset F^{k+j}$, and $\mathcal{R}=\oplus_{k \geq 0} F^{k} \cdot T^{k} \subset A[T]$ is a finitely generated $A$-algebra. Here $T$ is an indeterminate. There is a positive integer $N$ such that $F^{k N}=\left(F^{N}\right)^{k}$ holds for $k \geq 0$, and we assume that $F^{N}$ is $m$-primary. $G=\oplus_{k \geq 0} F^{k} / F^{k+1}$ and $G_{+}=\oplus_{k \geq 1} F^{k} / F^{k+1}$ as usual. The graded ring $G$ is also denoted by $\operatorname{gr}_{F}(A)$. We set $d=\operatorname{dim} A$. Our first result is the following.

\section{Theorem A.}

(1) Let a system of elements $x_{1}, \ldots, x_{s} \in G_{+}$be a minimal homogeneous generator system of $G_{+}$with $\operatorname{deg} x_{1} \leq \operatorname{deg} x_{2} \leq \cdots \leq \operatorname{deg} x_{s}$ with $s \geq d=$ $\operatorname{dim} A=\operatorname{dim} G$. Then we have the following:

$$
\begin{aligned}
\left(\prod_{i=1}^{d} \operatorname{deg} x_{i}\right) \lim _{\lambda \rightarrow 1}(1-\lambda)^{d} P(G, \lambda) & \leq{ }_{(i)} e(m, A) \\
& \leq e\left(G_{+}, G\right) \\
& \leq{ }_{(i i)}\left(\operatorname{deg} x_{s}\right)^{d} \lim _{\lambda \rightarrow 1}(1-\lambda)^{d} P(G, \lambda)
\end{aligned}
$$

where $P(G, \lambda)=\sum_{k \geq 0} l\left(G_{k}\right) \lambda^{k} \in \mathbf{Z}[[\lambda]]$.

(2) If the equality holds in (i), then $e(m, A)=e\left(G_{+}, G\right)$ and there is a parameter system $y_{1}, \ldots, y_{d}$ of $A$ whose initial form gives a homogeneous parameter system in $\left(y_{1}\right), \ldots$, in $\left(y_{d}\right)$ of $G$ such that $\operatorname{deg} i n\left(y_{i}\right)=\operatorname{deg} x_{i}$ for $i=1, \ldots, d$. Conversely, if there exists such a parameter system $y_{1}, \ldots, y_{d}$ of $A$, then these three integers coincide.

(3) If the equality holds in (ii) and $G$ is reduced, then $e(m, A)=e\left(G_{+}, G\right)$ and $G$ is a homogeneous ring. That is $\operatorname{deg} x_{1}=\cdots=\operatorname{deg} x_{s}$.

As an excellent and an important application of our theory, we study the simple K3 singularities of multiplicity two. The simple K3 singularity is a 
class of 3-dimensional complex analytic isolated Gorenstein singularity defined as a 3-dimensional analogue of simple elliptic singularity by S. Ishii and Kimio Watanabe [10] as a 3-dimensional Gorenstein isolated purely elliptic singularity of the Hodge type $(0,2)[6]$, [7], [22]. Related to the geometric characterization in [10], we can also characterize this class by the existence of a filtration of ideals as follows [19]:

Theorem-Definition (cf. Theorem 4.2 of [19]). $\quad$ Let $(A, m)$ be the local ring of a 3-dimensional Gorenstein isolated singularity. Then $(A, m)$ is a simple K3 singularity if and only if there is a filtration of ideals $F=\left\{F^{k}\right\}$ as above such that $\operatorname{gr}_{F}(A)$ is isomorphic to the Demazure construction $R(E, D)$ for some normal K3 surface $E$ with rational double points and ample integral Weil divisor $D$.

The filtration stated above is unique and the filtered blowing-up induces the canonical model of a resolution of singularity. We call this "the canonical filtration" of a simple K3 singularity.

In this situation, our main result is stated as follows:

Theorem B. Let $(A, m)$ be a simple $K 3$ singularity of multiplicity two, and $F$ the canonical filtration. Then $\operatorname{gr}_{F}(A)$ is also a hypersurface of multiplicity two.

In particular, $F$ is induced by a weight filtration on the coordinates of a suitable minimal embedding of $(A, m)$. In (7.1), Theorem $\mathrm{B}$ is proven as a corollary of Theorem A and the next theorem.

Theorem C. Let $E$ be a normal projective surface such that $\omega_{E} \cong O_{E}$ and $\mathrm{H}^{1}\left(E, O_{E}\right)=0$ and assume that $E$ has only rational double points. Let $D$ be an ample Q-Cartier integral Weil divisor and $G$ the normal graded ring represented by Demazure's construction $G=R(E, D)$. Let $x_{1}, \ldots, x_{s}$ be a minimal homogeneous generator of the homogeneous maximal ideal $G_{+}$with $\operatorname{deg} x_{1} \leq \cdots \leq \operatorname{deg} x_{s}$. Then we have the inequality $\operatorname{deg} x_{1} \cdot \operatorname{deg} x_{2} \cdot \operatorname{deg} x_{3} D^{2} \geq 2$.

Further as a corollary of the proof of Theorem C, we can classify the cases where the equality holds. There are exactly 48 types, which are listed in Table (7.3). By Theorem B and its corollaries, we can define the type of simple K3 double point by weighted homogeneous type of the initial form. They are 48 classes of (7.3) as same as quasi-homogeneous isolated singularities. Furthermore we prove that the weight type of the singularity stays the same under 
arbitrary one-parameter (FG)-deformations (Theorem (7.5)). These corollaries are shown in Section 7.

In [9], we found simple K3 singularities of multiplicity three where $G$ are not hypersurface. Hence we can not expect Theorem B for a simple K3 singularities of multiplicity three. For results about classification of quasi-homogeneous (or nondegenerate) simple K3 hypersurface singularities, we refer Yonemura's results [23]. Here this case is classified into the 95 weight type, which was originally discovered by M. Reid and by Fletcher [2].

In this paper, we assume that the local ring $(A, m)=\left(O_{V, p}, m\right)$ is coming from some scheme over an infinite field and analytically unramified.

\section{Chapter I. Multiplicity of Filtered Rings}

\section{$\S 1$. A Proof of Theorem A}

(1.1) As noted in Introduction, our singularity $(V, p)$ or local ring $(A, m)=$ $\left(O_{V, p}, m\right)$ is always coming from some scheme over an infinite field and analytically unramified. A filtration $F=\left\{F^{k}\right\}_{k>0}$ is a decreasing sequence of ideals of $A$ which satisfies; $F^{0}=A, F^{1}=m, F^{k} \supset F^{k+1}, F^{k} \cdot F^{j} \subset F^{k+j}$, and $\mathcal{R}=\oplus_{k \geq 0} F^{k} \cdot T^{k} \subset A[T]$ is a finitely generated $A$-algebra. Here $T$ is an indeterminate. There is a positive integer $N$ such that $F^{k N}=\left(F^{N}\right)^{k}$ holds for $k \geq 0$, and we assume that $F^{N}$ is $m$-primary. $G=\oplus_{k \geq 0} F^{k} / F^{k+1}$ and $G_{+}=\oplus_{k \geq 1} F^{k} / F^{k+1}$ as usual. We set $d=\operatorname{dim} A$. See [20] for a general information for the theory of filtered rings and filtered blowing-up.

First we shall prepare some lemmas.

Lemma 1.2. Let the situation be as above. Then

$$
l\left(A / m^{l+1}\right) \leq l\left(G /\left(G_{+}\right)^{l+1}\right) \quad \text { for } \quad l \geq 0 .
$$

In particular we obtain the relations $e(m, A) \leq e\left(G_{+}, G\right)$ and embdim $A \leq$ embdim $G$.

Proof. The filtration on $A / m^{l+1}$ induced by $F=\left\{F^{k}\right\}$ is given as follows:

$$
0 \rightarrow m^{l+1} \cap F^{k} \rightarrow F^{k} \rightarrow F^{k}\left(A / m^{l+1}\right) \rightarrow 0
$$

Hence we obtain $g r_{F}\left(A / m^{l+1}\right)=g r_{F}(A) / g r_{F}\left(m^{l+1}\right)$. Here we see

$$
g r_{F}\left(m^{l+1}\right)=\oplus_{k \geq 0} m^{l+1} \cap F^{k} / m^{l+1} \cap F^{k+1} \cong \oplus_{k \geq 0} \frac{F^{k} \cap m^{l+1}+F^{k+1}}{F^{k+1}},
$$




$$
\left(G_{+}\right)^{l+1}=\oplus_{k \geq l+1} \frac{\sum_{m_{1}+\cdots+m_{l+1}=k, m_{i} \geq 1} F^{m_{1}} \ldots F^{m_{l+1}}+F^{k+1}}{F^{k+1}} .
$$

Clearly we have

$$
\sum_{m_{1}+\cdots+m_{l+1}=k, m_{i} \geq 1} F^{m_{1}} \ldots F^{m_{l+1}} \subset F^{k} \cap m^{l+1} .
$$

Hence $\left(G_{+}\right)^{l+1} \subset g r_{F}\left(m^{l+1}\right)$. Therefore

$$
l\left(A / m^{l+1}\right)=l\left(g r_{F}\left(A / m^{l+1}\right)\right)=l\left(G / g r_{F}\left(m^{l+1}\right)\right) \leq l\left(G /\left(G_{+}\right)^{l+1}\right) .
$$

Lemma 1.3. Let $L$ be a positive integer such that the relation $F^{m L}=$ $\left(F^{L}\right)^{m}$ holds for any positive integer $m$. Then

$$
e\left(F^{L}, A\right)=L^{d} \cdot \lim _{\lambda \rightarrow 1}(1-\lambda)^{d} P(G, \lambda) .
$$

Proof. By the assumption, $\oplus_{k \geq 0} F^{k L} / F^{(k+1) L}$ is generated by $F^{L} / F^{2 L}$. Hence we obtain the equality (see Sections 13 and 14 of [12]):

$$
e\left(F^{L}, A\right)=\lim _{\lambda \rightarrow 1}(1-\lambda)^{d} P\left(\oplus_{k \geq 0} F^{k L} / F^{(k+1) L}, \lambda\right) .
$$

Let $G^{(L, l)}=\oplus_{k \geq 0} F^{k L+l} / F^{k L+l+1}$ for $l=0, \ldots, L-1$. Since there is an integer $M$ such that $F^{L} \cdot F^{b}=F^{L+b}$ holds for any $b \geq M, G^{(L, l)}$ is a finite $G^{(L, 0)}$-module for $l=0, \ldots, L-1$. As graded $G^{(L, 0)}$-modules, we calculate the Poincare series; $P\left(G^{(L, l)}, \mu\right) \in \mathbf{Z}[[\mu]]$ for $l=0, \ldots, L-1$. For each $l$, $\lim _{\mu \rightarrow 1}(1-\mu)^{d} P\left(G^{(L, l)}, \mu\right)$ is a finite number. Hence

$$
\begin{aligned}
\lim _{\mu \rightarrow 1}(1-\mu)^{d} P(G, \mu) & =\lim _{\mu \rightarrow 1}\left(1-\mu^{L}\right)^{d} \sum_{l=0}^{L-1} P\left(G^{(L, l)}, \mu^{L}\right) \mu^{l} \cdot \frac{(1-\mu)^{d}}{\left(1-\mu^{L}\right)^{d}} \\
& =\lim _{\nu \rightarrow 1}(1-\nu)^{d} \sum_{l=0}^{L-1} P\left(G^{(L, l)}, \nu\right) \cdot \frac{1}{L^{d}} \\
& =\lim _{\nu \rightarrow 1}(1-\nu)^{d} P\left(\oplus_{k \geq 0} F^{k L} / F^{(k+1) L}, \nu\right) \cdot \frac{1}{L^{d}} \\
& =e\left(F^{L}, A\right) \frac{1}{L^{d}} .
\end{aligned}
$$

Next we recall a theorem of C. P. Ramanujan. We may assume that $p$ is a closed point of a projective variety $\bar{V}$ over the field $k$. Let $I$ be an $m$-primary ideal of $O_{V, p}$. Let $\pi: \tilde{V} \rightarrow V$ be a projective morphism such that $I . O_{\tilde{V}}$ is a locally principal $O_{\tilde{V}}$-module. Representing $I . O_{\tilde{V}}=O_{\tilde{V}}(-D(I, \pi))$ by a Cartier divisor on $\tilde{V}$, a theorem of C. P. Ramanujan on the multiplicity $e\left(I, O_{V, p}\right)$ is given as; 
Lemma $1.4([13]) . \quad e\left(I, O_{V, p}\right)=(-1)^{d+1} D(I, \pi)^{d}$. Here $d=\operatorname{dim} O_{V, p}$.

Lemma 1.5. Let $z_{1}, \ldots, z_{d} \in G_{+}$be a homogeneous parameter system of $G$. Then we have

$$
e\left(\left(z_{1}, \ldots, z_{d}\right), G\right)=\left(\prod_{i=1}^{d} \operatorname{deg} z_{i}\right) \lim _{\lambda \rightarrow 1}(1-\lambda)^{d} P(G, \lambda) .
$$

Proof. We introduce a natural filtration on $G$ by the grading as follows: $F^{k}(G)=\left.G\right|_{k}=\oplus_{l \geq k} G_{l} \subset G$ for $k \in \mathbf{Z}$. Here $\left.\oplus_{k \geq 0} G\right|_{k} U^{k} \subset G[U]$ is a finitely generated $G$-algebra, where $U$ is an indeterminate. Let $C=$ $\operatorname{Proj}\left(\left.\oplus_{k \geq 0} G\right|_{k} U^{k}\right) \longrightarrow \operatorname{Spec}(G)$ be the natural blowing-up by this grading. There is an integer $L$ where the relation $\left.G\right|_{L m}=\left(\left.G\right|_{L}\right)^{m}$ holds for $m \in \mathbf{N}$. Then on $C$ we have $\left.G\right|_{L} O_{C}=O_{C}(L)=G \tilde{(L)} \subset O_{C}$ and this is an invertible $O_{C}$-module sheaf. We can choose $L \in \mathbf{N}$ such that $\operatorname{deg} z_{i} \mid L$ for $i=1, \ldots, d$. Then $\left(z_{1}^{L / \operatorname{deg} z_{1}}, \ldots, z_{d}^{L / \operatorname{deg} z_{d}}\right)$ is a parameter of $G$ and one can easily check the relation

$$
\left(z_{1}^{L / \operatorname{deg} z_{1}}, \ldots, z_{d}^{L / \operatorname{deg} z_{d}}\right) O_{C}=O_{C}(L) .
$$

By Ramanujan's Theorem 1.4, Lech's lemma (Theorem 14.12 [12]) and Lemma 1.3 , we obtain the relations

$$
\begin{aligned}
\frac{L}{\operatorname{deg} z_{1}} \ldots \cdot \frac{L}{\operatorname{deg} z_{d}} \cdot e\left(\left(z_{1}, \ldots, z_{d}\right), G\right) & =e\left(\left(z_{1}^{\frac{L}{\operatorname{deg} z_{1}}}, \ldots, z_{d}^{\frac{L}{\operatorname{deg} z_{d}}}\right), G\right) \\
& =e\left(\left.G\right|_{L}, G\right)=L^{d} \cdot \lim _{\lambda \rightarrow 1}(1-\lambda)^{d} P(G, \lambda) .
\end{aligned}
$$

(1.6) Proof of the inequality (i) in (1). Let a system of elements $x_{1}, \ldots, x_{s}$ of the maximal ideal $m$ of $A$ whose initial forms with respect to the filtration $F$ give the minimal homogeneous generator of $G_{+}$as follows; $x_{i} \in F^{q_{i}}-F^{q_{i}+1}$ and the initial forms $i n_{F}\left(x_{i}\right)=\bar{x}_{i} \in G_{q_{i}}$ satisfies the relations $G_{+}=\left(\bar{x}_{1}, \ldots, \bar{x}_{s}\right) G$ and $q_{1} \leq \cdots \leq q_{s}$. We can easily see the relations $m=F^{n}+\left(x_{1}, \ldots, x_{s}\right) A$ for any positive integer $n$. There is an integer $n$ such that $F^{n} \subset m^{2}$. Hence $m=\left(x_{1}, \ldots, x_{s}\right)$ by NAK. There is a system of parameter $y_{1}, \ldots, y_{d}$ which is a minimal reduction of $m$ and given as linear combination of $x_{1}, \ldots, x_{s}$ as follows: $y_{i}=\sum_{j=1}^{s} a_{i, j} x_{j}$, (where $a_{i, j} \in k$ ) with $1 \leq i \leq d, 1 \leq j \leq s$. By the proof of Theorem 14.14 [12] (see pp. 113-114), there is a Zariski open set $U$ of $k^{s d}$ where $\left(y_{1}, \ldots, y_{d}\right)$ in the above is a reduction of $m$ for $\left(a_{i, j}\right) \in U$. In particular, there is a reduction in the above form with $\operatorname{det}\left(a_{i, j}\right)_{1 \leq i, j \leq d} \neq 0$. Hence we can choose a reduction $\left(y_{1}, \ldots, y_{d}\right)$ in the following form:

$$
y_{i}=x_{i}+\sum_{j=d+1}^{s} a_{i, j} x_{j} \quad \text { where } \quad a_{i, j} \in k
$$


for $1 \leq i \leq d$ from the beginning.

Let $L$ be a positive integer divided by L.C.M. $\left(\operatorname{deg} x_{1}, \ldots, \operatorname{deg} x_{d}\right)$. By Lech's lemma (Theorem 14.12 [12])

$$
e\left(\left(y_{1}^{\frac{L}{q_{1}}}, \ldots, y_{d}^{\frac{L}{q_{d}}}\right), A\right)=\frac{L}{q_{1}} \ldots \cdot \frac{L}{q_{d}} \cdot e\left(\left(y_{1}, \ldots, y_{d}\right), A\right) .
$$

Since $y_{i}^{L / q_{i}} \in F^{L}$ for $1 \leq i \leq d$, we have

$$
e\left(F^{L}, A\right) \leq \frac{L}{q_{1}} \ldots \cdot \frac{L}{q_{d}} \cdot e\left(\left(y_{1}, \ldots, y_{d}\right), A\right) .
$$

There is an integer $L$ as above and satisfies the relation $F^{m L}=\left(F^{L}\right)^{m}$ for any positive integer $m$. By Lemmas 1.3 and 1.5, we obtain the assertion.

(1.7) Proof of (2). Let $y_{1}, \ldots, y_{d}$ be a parameter system of $A$ as in the arguments of (1.6). First we assume the equality (i) holds. By the arguments in (1.6), we have the equality

$$
e\left(\left(y_{1}^{\frac{L}{q_{1}}}, \ldots, y_{d}^{\frac{L}{q_{d}}}\right), A\right)=e\left(F^{L}, A\right)
$$

By a Theorem of Rees ([16], [5]), $\left(y_{1}^{L / q_{1}}, \ldots, y_{d}^{L / q_{d}}\right)$ is a reduction of $F^{L}$. That is there is an integer $r>0$ such that $\left(F^{L}\right)^{r+1}=\left(F^{L}\right)^{r}\left(y_{1}^{L / q_{1}}, \ldots, y_{d}^{L / q_{d}}\right)$ in $A$. Let $\psi: X=\operatorname{Proj}\left(\oplus_{k \geq 0} F^{k} . T^{k}\right) \rightarrow \operatorname{Spec}(A)$ the filtered blowing-up of $\operatorname{Spec}(A)$ by $F$. On $X$ this leads the relation

$$
\left(F^{L}\right)^{r+1} O_{X}=\left(F^{L}\right)^{r}\left(y_{1}^{\frac{L}{q_{1}}}, \ldots, y_{d}^{\frac{L}{q_{d}}}\right) O_{X} \quad \text { in } \quad O_{X} .
$$

Here $F^{L} O_{X}=O_{X}(L)$ is an invertible $O_{X}$-module sheaf. Hence we obtain the relation

$$
F^{L} O_{X}=O_{X}(L)=\left(y_{1}^{\frac{L}{q_{1}}}, \ldots, y_{d}^{\frac{L}{q_{d}}}\right) O_{X}
$$

We represent the strict transform of the scheme $\operatorname{Spec}\left(A / y_{i}\right)$ by $\psi$ as $W_{y_{i}, \psi}$ for $i=1, \ldots, d$. Since $\left(y_{1}^{L / q_{1}}, \ldots, y_{d}^{L / q_{d}}\right) O_{X}$ is base point free, $W_{y_{1}, \psi} \cap \cdots \cap$ $W_{y_{d}, \psi} \cap E$ is empty. Here $W_{y_{j}, \psi} \cap E=\operatorname{Proj}\left(G / i n\left(y_{j}\right) G\right)$, where $i n\left(y_{j}\right)$ is the initial homogeneous element of $y_{j}$. Therefore $i n\left(y_{1}\right), \ldots, i n\left(y_{d}\right)$ is a parameter system of $G$. By Lemma 1.5, we obtain the relation

$$
\begin{aligned}
e\left(G_{+}, G\right) & \leq e\left(\left(\operatorname{in}\left(y_{1}\right), \ldots, i n\left(y_{d}\right)\right), G\right) \\
& =\left(\prod_{i=1}^{d} \operatorname{deg} x_{i}\right) \lim _{\lambda \rightarrow 1}(1-\lambda)^{d} P(G, \lambda)=e(m, A) .
\end{aligned}
$$


By Lemma 1.2 these are the same.

The proof of the converse implication follows from Lemma 1.5.

(1.8) Proof of the inequality (ii) of (1). As in (1.5), there is an integer $L$ satisfies the relation $\left.G\right|_{m L}=\left(\left.G\right|_{L}\right)^{m}$ and we have $e\left(\left.G\right|_{L}, G\right)=L^{d} \cdot \lim _{\lambda \rightarrow 1}(1-$ $\lambda)^{d} P(G, \lambda)$ by (1.3). We can easily see $\left.G\right|_{q_{s} L} \subset\left(G_{+}\right)^{L}$. Hence

$$
\begin{aligned}
L^{d} e\left(G_{+}, G\right)=e\left(\left(G_{+}\right)^{L}, G\right) & \leq e\left(\left.G\right|_{q_{s} L}, G\right)=e\left(\left(\left.G\right|_{L}\right)^{q_{s}}, G\right)=q_{s}^{d} e\left(\left.G\right|_{L}, G\right) \\
& =q_{s}^{d} \cdot L^{d} \cdot \lim _{\lambda \rightarrow 1}(1-\lambda)^{d} P(G, \lambda)
\end{aligned}
$$

Therefore $e\left(G_{+}, G\right) \leq q_{s}^{d}$. $\lim _{\lambda \rightarrow 1}(1-\lambda)^{d} P(G, \lambda)$.

(1.9) Proof of (3). Assume that the equality $e\left(G_{+}, G\right)=q_{s}^{d} \cdot \lim _{\lambda \rightarrow 1}(1-$ $\lambda)^{d} P(G, \lambda)$ holds. In the arguments of $(1.8)$, we have the relation $e\left(\left(G_{+}\right)^{L}, G\right)=$ $e\left(\left.G\right|_{q_{s} L}, G\right)$. We can choose a sufficiently large $L$ where $O_{C}\left(q_{s} L\right)=\left.G\right|_{q_{s} L} O_{C} \subset$ $O_{C}$ is an invertible $O_{C}$-module sheaf. By a theorem of Rees ([16], [5]), $\left.G\right|_{q_{s} L}$ is a reduction of $G_{+}^{L}$. As in (1.5) we obtain the relation $G_{+}^{L} O_{C}=O_{C}\left(q_{s} L\right) \subset$ $O_{C}\left(q_{1} L\right)$. We will show $q_{1}=q_{s}$. Otherwise $q_{1}<q_{s}$. Then $0 \neq x_{1}^{L} \in$ $G_{q_{1} L}$, hence $\left.x_{1}^{L} \in G\right|_{q_{1} L}-\left.G\right|_{q_{1} L+1}$ and $q_{1} L+1 \leq q_{s} L$. On $D_{+}\left(\left(x_{1}^{L}\right)^{*}\right)$, $\left.O_{C}\left(q_{1} L\right)\right|_{D_{+}\left(\left(x_{1}^{L}\right)^{*}\right)}=\left.x_{1}^{L} O_{C}\right|_{D_{+}\left(\left(x_{1}^{L}\right)^{*}\right)}$ and $x_{1}^{L} O_{C} \not \subset O_{C}\left(q_{1} L+1\right)$ (cf. [20] for a generality of filtered blowing). Hence $G_{+}^{L} O_{C} \subset \neq O_{C}\left(q_{s} L\right)$. Therefore we obtain the relation $q_{1}=q_{s}$. Now we set this integer by $q$. Then we obtain the relations $F^{k}=m^{[(k+q-1) / q]}$ for $k \geq 0$ and $G \cong g r_{m}(A)$ as rings. Therefore, we obtain $e\left(G_{+}, G\right)=e(m, A)$.

\section{§2. Remarks on Normal Graded Rings}

The purpose of this section is to collect the generalities of the number $\left(\prod_{i=1}^{d} \operatorname{deg} x_{i}\right) \lim _{\lambda \rightarrow 1}(1-\lambda)^{d} P(G, \lambda)$ in the case $G$ is normal and represented by Demazure's construction $R(E, D)=G$. Please refer [1], [21] for the basic facts on Demazure's construction of normal graded rings. The following proposition is a joint work with K.-i. Watanabe.

Proposition 2.1. $\quad$ Let $R=R(E, D)$ be a normal d-dimensional graded ring with Demazure's description. Then we have the following.

$$
D^{d-1}=\lim _{\lambda \rightarrow 1}(1-\lambda)^{d} P(R, \lambda)
$$

where $P(R, \lambda)=\sum_{k \geq 0} l\left(R_{k}\right) \lambda^{k} \in \mathbf{Z}[[\lambda]]$, with $d=\operatorname{dim} R$.

Proof. If $R$ is generated by $R_{1}$, then $D$ is Cartier. Then we have $e\left(R_{+}, R\right)=\lim _{\lambda \rightarrow 1}(1-\lambda)^{d} P(R, \lambda)[12]$, and the assertion $e\left(R_{+}, R\right)=D^{d-1}$ 
is standard. Now we will show the assertion in general. Let $N$ be a positive integer such that $R^{(N)}=R(E, N D)$ is generated by $R_{N}$. Let $x_{1}, \ldots, x_{d}$ be a homogeneous parameter system of $R$. Then $x_{1}^{N}, \ldots, x_{d}^{N}$ is a parameter of $R^{(N)}$. Hence we have $N^{d} e\left(\left(x_{1}, \ldots, x_{d}\right), R\right)=e\left(\left(x_{1}^{N}, \ldots, x_{d}^{N}\right), R\right)=\operatorname{rank}_{R^{(N)}} R$ $\cdot e\left(\left(x_{1}^{N}, \ldots, x_{d}^{N}\right), R^{(N)}\right)$. By (1.5), $e\left(\left(x_{1}^{N}, \ldots, x_{d}^{N}\right), R^{(N)}\right)=\left(\prod_{i=1}^{d} \operatorname{deg} x_{i}\right)$ $\times \lim _{\lambda \rightarrow 1}(1-\lambda)^{d} P\left(R^{(N)}, \lambda\right)=\left(\prod_{i=1}^{d} \operatorname{deg} x_{i}\right)(N D)^{d-1}$. Here remark that the degree of $x_{i}^{N}$ in $R^{(N)}$ is $\operatorname{deg} x_{i}$. Hence $N^{d} e\left(\left(x_{1}, \ldots, x_{d}\right), R\right)=N^{d}\left(\prod_{i=1}^{d} \operatorname{deg} x_{i}\right)$ $D^{d-1}$ follows. On the other hand we have the relation $(1.5): e\left(\left(x_{1}, \ldots, x_{d}\right), R\right)$ $=\left(\prod_{i=1}^{d} \operatorname{deg} x_{i}\right) \lim _{\lambda \rightarrow 1}(1-\lambda)^{d} P(R, \lambda)$. This completes the proof.

Example 2.2. For a graded complete intersection

$$
R=k\left[x_{1}, \ldots, x_{d+s}\right] /\left(f_{1}, \ldots, f_{s}\right),
$$

where $f_{1}, \ldots, f_{s}$ is a homogeneous regular sequence of $k\left[x_{1}, \ldots, x_{d+s}\right]$, we have

$$
P(R, \lambda)=\frac{\left(1-\lambda^{\operatorname{deg} f_{1}}\right) \ldots \cdot\left(1-\lambda^{\operatorname{deg} f_{s}}\right)}{\left(1-\lambda^{\operatorname{deg} x_{1}}\right) \ldots\left(1-\lambda^{\operatorname{deg} x_{d+s}}\right)} .
$$

Hence

$$
\lim _{\lambda \rightarrow 1}(1-\lambda)^{d} P(R, \lambda)=\frac{\left(\operatorname{deg} f_{1}\right) \ldots \ldots\left(\operatorname{deg} f_{s}\right)}{\left(\operatorname{deg} x_{1}\right) \ldots\left(\operatorname{deg} x_{d+s}\right)} .
$$

If $R$ is normal and represented as $R=R(E, D)$, then (2.1) implies the following the relation

$$
D^{d-1}=\frac{\left(\operatorname{deg} f_{1}\right) \ldots\left(\operatorname{deg} f_{s}\right)}{\left(\operatorname{deg} x_{1}\right) \ldots\left(\operatorname{deg} x_{d+s}\right)} .
$$

What information determines this number? Next we prove the following lemma to obtain Corollary 2.4.

Lemma 2.3. Let a system of homogeneous elements $x_{1}, \ldots, x_{s}$ of $G$ be a minimal generator of the homogeneous maximal ideal $G_{+}$as same as in Theorem A. If $G$ is a normal domain over an algebraically closed field $k=G_{0}$, then any couple $x_{i}$ and $x_{j}$ with $i \neq j$ are algebraically independent over $k$.

Proof (Based on the idea due to Kyoji Saito). We will show this by a contradiction. Suppose that $x_{i}$ and $x_{j}$ is algebraically dependent over $k=G_{0}$ for some pair $i, j$ with $i \neq j$. There is a weighted homogeneous irreducible polynomial $P(s, t) \in k[s, t]$ such that

$$
k[s, t] / P=k\left[x_{i}, x_{j}\right] \subset G .
$$


Here $k\left[x_{i}, x_{j}\right] \cap G_{+}$is the homogeneous maximal ideal of $k\left[x_{i}, x_{j}\right]$. By taking the completions we obtain the following

$$
k[[s, t]] / P=k\left[\left[x_{i}, x_{j}\right]\right] \subset G^{\wedge} .
$$

Here $x_{i}, x_{j} \in G_{+} \cdot G^{\wedge}$. Since $G$ is essentially of finite type over $k, G^{\wedge}$ is also a normal domain. There exists $u \in G_{+} \cdot G^{\wedge}$ where the normalization of $k\left[\left[x_{i}, x_{j}\right]\right]$ is written as $k[[u]]$ and we have

$$
k\left[\left[x_{i}, x_{j}\right]\right] \subset k[[u]] \subset G^{\wedge} .
$$

There are $a, b \in k$ such that

$$
x_{i}-a \cdot u, \quad x_{j}-b \cdot u \in u^{2} k[[u]] \subset G_{+}^{2} \cdot G^{\wedge} .
$$

Hence

$$
a \cdot x_{j}-b \cdot x_{i} \in G_{+}^{2} \cdot G^{\wedge} \cap G=G_{+}^{2} .
$$

Since $x_{i}$ and $x_{j}$ are linearly independent in $G_{+} / G_{+}^{2}$, we have $a=b=0$. Hence $x_{i} \in G_{+}^{2} \cdot G^{\wedge} \cap G=G_{+}^{2}$. This is a contradiction.

Corollary 2.4. Let $x_{1}, \ldots, x_{s}$ be a minimal generator of the homogeneous maximal ideal $G_{+}$as same as in Theorem A. If $G$ is a normal domain over an algebraically closed field $k=G_{0}$, then the lower first three degrees $\operatorname{deg} x_{1}, \operatorname{deg} x_{2}, \operatorname{deg} x_{3}$ are completely determined from the Poincare-Hilbert series $P(G, \lambda)$. In particular, in the case $\operatorname{dim} G \leq 3$,

$$
\left(\prod_{i=1}^{d} \operatorname{deg} x_{i}\right) \lim _{\lambda \rightarrow 1}(1-\lambda)^{d} P(G, \lambda)
$$

is determined by $P(G, \lambda)$.

The following example says that $e(m, A)$ and $e\left(G_{+}, G\right)$ are different, in general, even if we assume that $G$ is a normal Gorenstein domain.

Example 2.5. We introduce the filtration $F$ on the local ring $A$ as follows:

(i) Let $A=k[[x, y, z, w]] /\left(w-x^{2}-y^{2}-z^{2}\right) \cong k[[x, y, z]]$ and $F$ the induced filtration from the filtration on $k[[x, y, z, w]]$ by the monomial degrees as $F^{k}=\left\{x^{a} y^{b} z^{c} w^{d} \in k[x, y, z, w] \mid a+b+c+3 d \geq k\right\} A$. One can easily see that

$$
G=g r_{F}(A) \cong k[x, y, z, w] / x^{2}+y^{2}+z^{2}
$$


Theorem A says

$$
\text { 1.1.1. } \lim _{\lambda \rightarrow 1}(1-\lambda)^{d} P(G, \lambda)=1.1 .1 \cdot \frac{2}{1.1 .1 .3} \leq 1=e(m, A) \leq e\left(G_{+}, G\right)=2 .
$$

(ii) Let $A=k[[u, x, y, z, w]] /\left(u+y^{3}+z^{7}+w^{21}, x^{2}-u w\right) \cong k[[x, y, z, w]] /\left(x^{2}+\right.$ $\left.w\left(y^{3}+z^{7}+w^{21}\right)\right)$ and $F$ the induced filtration from the filtration on $k[[u, x, y, z, w]]$ by the monomial degrees as $F^{k}=\left\{u^{\alpha} x^{\beta} y^{\gamma} z^{\delta} w^{\varepsilon} \in\right.$ $k[u, x, y, z, w] \mid 23 \alpha+12 \beta+7 \gamma+3 \delta+\varepsilon \geq k\} A$. One can easily see that

$$
G=g r_{F}(A) \cong k[u, x, y, z, w] /\left(y^{3}+z^{7}+w^{21}, x^{2}-u w\right) .
$$

Theorem A says

$$
\text { 1.3.7. } \begin{aligned}
\lim _{\lambda \rightarrow 1}(1-\lambda)^{d} P(G, \lambda) & =1.3 .7 \cdot \frac{21.24}{1.3 .7 .12 .23} \leq 2 \\
& =e(m, A) \leq e\left(G_{+}, G\right)=6 .
\end{aligned}
$$

Corollary 2.6. $\quad$ Let the situation be as in Theorem A.

(1) If the condition

the round up of the number $\left(\prod_{i=1}^{d} \operatorname{deg} x_{i}\right) \lim _{\lambda \rightarrow 1}(1-\lambda)^{d} P(G, \lambda)=e\left(G_{+}, G\right)$ holds, then the equality $e(m, A)=e\left(G_{+}, G\right)$ holds.

(2) If $G$ is a hypersurface with the isolated singularity at $G_{+}$, then $e(m, A)=$ $e\left(G_{+}, G\right)$.

Proof. (1) is obvious from (1) of Theorem A. (2) Let us represent $G$ as $G=k\left[x_{1}, \ldots, x_{d+1}\right] / f$ by a weighted homogeneous polynomial $f$ of the type $\left(q_{1}, \ldots, q_{d+1} ; h\right)$ with $\operatorname{deg} x_{i}=q_{i}$ and $q_{1} \leq \cdots \leq q_{d+1}$. By $(2.2)$ and Theorem A, we have $h /\left(q_{d+1}\right)=q_{1} \ldots q_{d} \lim _{\lambda \rightarrow 1}(1-\lambda)^{d} P(G, \lambda) \leq e\left(G_{+}, G\right)=\operatorname{ord} f$. Since $\{f=0\}$ has only isolated singularity at $o$, a monomial of form $x_{i}^{m_{i}} x_{j(i)}$ with $j(i) \in\{1, \ldots, d+1\}$ appears in $f$ with non-zero coefficients for each $i$ (K. Saito [17]). This implies ord $f \leq m_{d+1}+1<h / q_{d+1}+1$. Hence $e\left(G_{+}, G\right)$ equals the round up of the rational number $h / q_{d+1}$.

\section{Chapter II. The Inequality $q_{1} q_{2} q_{3} D^{2} \geq 2$ for Normal K3 Surfaces}

The rest of this paper is devoted to the proof of Theorem C. From here we will set $q_{i}=\operatorname{deg} x_{i}$ by simplicity. Our basic strategy is as follows: By 
Corollary 2.4, the behavior of $b_{m}=\operatorname{dim} H^{0}\left(E, O_{E}(m D)\right)$ determines $q_{1}, q_{2}, q_{3}$. Then Reid's singular Riemann-Roch formula for surfaces with rational double points is very effective. In fact, using (3.4.1), we can see that first $4 b_{m}$ determines the essential information on baskets in many cases. As a corollary of our proof, we can classify the data $\left\{D^{2}\right.$, baskets of singularities $\}$ where the relation $q_{1} q_{2} q_{3} D^{2}=2$ holds.

\section{$\S 3 . \quad$ Some Easy Cases}

In this section, we will derive convenient formulas from Reid's RiemannRoch formula for normal surface and prove the assertion of Theorem $\mathrm{C}$ in some easy general cases. Now we recall the following famous formula.

Theorem 3.1 (M. Reid [15, (9.1)] ).

(I) There is a formula

$$
\chi\left(E, O_{E}(D)\right)=\chi\left(O_{E}\right)+\frac{1}{2}\left(D^{2}-D K_{E}\right)+\sum_{Q} c_{Q}(D)
$$

where $c_{Q}(D)=c_{Q}\left(O_{E}(D)\right) \in \mathbf{Q}$ is a contribution due to the singularity of $O_{E}(D)$ at $Q$, depending only on the local analytic type of $Q \in E$ and $D$; the sum takes place over the singularities of $D$ (the points $Q \in E$ at which $D$ is not Cartier).

(II) If $P \in E$ and $D$ is a cyclic quotient singularity of type ${ }_{i}((1 / r)(1,-1))$ then $c_{P}(D)=-(i(r-i)) / 2 r$.

(III) For every rational double singularity $Q \in E$ and Weil divisor $D$ on $E$, there exists a basket of points of $\left\{P_{\alpha} \in E_{\alpha}\right.$ and $\left.D_{\alpha}\right\}$ of type $i_{\alpha}\left(\left(1 / r_{\alpha}\right)(1,-1)\right)$ and with $i_{\alpha}$ coprime to $r_{\alpha}$, such that

$$
c_{Q}(D)=\sum_{\alpha} c_{P_{\alpha}}\left(D_{\alpha}\right)=-\sum_{\alpha} \frac{i_{\alpha}\left(r_{\alpha}-i_{\alpha}\right)}{2 r_{\alpha}} .
$$

Remark 3.2. (i) This theorem says that the correction terms for the Riemann-Roch formula from the rational double points can be counted by certain numbers of set of locally poralized singularities of $A_{r}$-types. Further concerning the polarization $i_{\alpha} \in\left\{1, \ldots, r_{\alpha}-1\right\}$ defined by $D$ in $\mathbf{Z} / r_{\alpha} \mathbf{Z} \cong$ $C l\left(A_{r_{\alpha}-1}\right)$, we may assume $2 i_{\alpha} \leq r_{\alpha}$ by the symmetry of the behavior of $\left(\overline{k i_{\alpha}}\left(r_{\alpha}-\overline{k i_{\alpha}}\right)\right) / 2 r_{\alpha}$ for $k \geq 0$. (ii) In the original proof of (III) of (3.1), M. Reid used some deformation arguments. We remark that this fact also is shown by other way, e.g. by using Giraud's version of Riemann-Roch formula [4]. 
(3.3) Let $E$ be a normal K3 surface and $D$ an ample integral divisor as in the situation of Theorem C. As in (3.2) (i), we assume the conditions $2 i_{\alpha} \leq r_{\alpha}$ for all $\alpha$ in the basket of singularities. Let $b_{m}=\operatorname{dim} H^{0}\left(E, O_{E}(m D)\right)$. Then, by Kawamata's vanishing theorem [11], we have

$$
b_{m}=\frac{m^{2} D^{2}}{2}+2+\sum_{Q} c_{Q}(m D) \quad \text { for } \quad m \geq 1 .
$$

Lemma 3.4. Assume the conditions $2 i_{\alpha} \leq r_{\alpha}$ for all $\alpha$. Then we have the following relations.

(1) $D^{2}=2\left(b_{1}-2\right)+\sum_{\alpha=1}^{N}\left(\left(i_{\alpha}\left(r_{\alpha}-i_{\alpha}\right)\right) / r_{\alpha}\right)$,

(2) $\sum_{\alpha=1}^{N} i_{\alpha}=b_{2}-4 b_{1}+6$,

(3) $b_{m}=m^{2}-3 m+2+\left(-m^{2}+2 m\right) b_{1}+\left(\left(m^{2}-m\right) / 2\right) b_{2}-\sum_{s \geq 1} \sum_{\alpha}\left(m i_{\alpha}\right.$ $\left.-s r_{\alpha}\right)_{+}$, where the symbol $\ell_{+}$is defined as $\ell_{+}=\max \{0, \ell\}$.

Proof. (1) is nothing but (3.3) for $m=1$, and we have $b_{1}=2+\left(D^{2} / 2\right)-$ $\sum_{\alpha}\left(\left(i_{\alpha}\left(r_{\alpha}-i_{\alpha}\right)\right) / 2 r_{\alpha}\right)$. By the assumption $2 i_{\alpha} \leq r_{\alpha}$, we have $b_{2}=2+2 D^{2}-$ $\sum_{\alpha}\left(\left(2 i_{\alpha}\left(r_{\alpha}-2 i_{\alpha}\right)\right) / 2 r_{\alpha}\right)$. Hence we obtain the relation $b_{2}-4 b_{1}=-6+\sum_{\alpha} i_{\alpha}$ and the assertion of (2) follows. We can also see the relation $D^{2}=2-2 b_{1}+b_{2}$ $-\sum_{\alpha}\left(\left(\left(i_{\alpha}\right)^{2}\right) / r_{\alpha}\right)$. By these, we obtain the relation $b_{m}=m^{2}-3 m+2+$ $\left(-m^{2}+2 m\right) b_{1}+\left(\left(m^{2}-m\right) / 2\right) b_{2}+\sum_{\alpha}\left(m i_{\alpha} r_{\alpha}-m^{2}\left(i_{\alpha}\right)^{2}-\overline{m i_{\alpha}} \cdot r_{\alpha}+\left(\overline{m i_{\alpha}}\right)^{2}\right) / 2 r_{\alpha}$. If $(\ell-1) r_{\alpha}<m i_{\alpha} \leq \ell r_{\alpha}$, then $\overline{m i_{\alpha}}=m i_{\alpha}-(\ell-1) r_{\alpha}$, and we can easily see the relation $\left(m i_{\alpha} r_{\alpha}-m^{2}\left(i_{\alpha}\right)^{2}-\overline{m i_{\alpha}} \cdot r_{\alpha}+\left(\overline{m i_{\alpha}}\right)^{2}\right) / 2 r_{\alpha}=-\left\{\left(m i_{\alpha}-r_{\alpha}\right)+\right.$ $\left.\left(m i_{\alpha}-2 r_{\alpha}\right)+\cdots+\left(m i_{\alpha}-(\ell-1) r_{\alpha}\right)\right\}$.

We start the proof of Theorem $\mathrm{C}$ by the following.

Lemma 3.5. Let $k \geq 2$ and assume that $b_{1}=b_{2}=\cdots=b_{k-1}=0$ and that $b_{k} \geq 2$. Then we obtain the inequality $q_{1} q_{2} q_{3} D^{2} \geq 2$. Further $q_{1} q_{2} q_{3} D^{2}=2$ holds if and only if $k=2, N=8,\left(i_{1}, \ldots, i_{8}\right)=(1, \ldots, 1)$, and $\left(r_{1}, \ldots, r_{8}\right)=$ $(2,2,2,2,2,2,2,3)$.

Proof. First assume that $b_{k} \geq 3$. Then $q_{1}=q_{2}=q_{3}=k$. By (3.3), we obtain the relations: $k^{2} D^{2}=2\left(b_{k}-2\right)+\sum_{\alpha}\left(\left(\overline{k i_{\alpha}}\left(r_{\alpha}-\overline{k i_{\alpha}}\right)\right) / r_{\alpha}\right) \geq 2\left(b_{k}-2\right) \geq 2$. Since $k \geq 2, q_{1} q_{2} q_{3} D^{2} \geq 4$ follows.

Now assume that $b_{k}=2$. By the arguments in the above, we have $q_{1}=$ $q_{2}=k, q_{3} \geq k+1$, and $0<k^{2} D^{2}=\sum_{\alpha}\left(\left(\overline{k i_{\alpha}}\left(r_{\alpha}-\overline{k i_{\alpha}}\right)\right) / r_{\alpha}\right)$. There is an index $\alpha_{0}$ such that $\overline{k i_{\alpha_{0}}} \neq 0$. We have the relation $q_{1} q_{2} q_{3} D^{2} \geq k \cdot k \cdot(k+1) D^{2} \geq$ $(k+1)\left(\left(r_{\alpha_{0}}-1\right) / r_{\alpha_{0}}\right) \geq(k+1) / 2$. 
Hence if $k \geq 4$, we obtain $q_{1} q_{2} q_{3} D^{2} \geq(5 / 2)>2$.

Next assume $k=3$. We have $b_{1}=b_{2}=0$ and $b_{3}=2-\sum_{\alpha=1}^{N}\left(3 i_{\alpha}-r_{\alpha}\right)_{+}$ by (3.4.3). The condition $b_{3}=2$ implies $3 \leq 3 i_{\alpha} \leq r_{\alpha}$ for all $\alpha$. Since $\overline{3 i_{\alpha_{0}}} \neq 0$, $r_{\alpha_{0}} \geq 4$. Hence, $q_{1} q_{2} q_{3} D^{2} \geq 4\left(\left(r_{\alpha_{0}}-1\right) / r_{\alpha_{0}}\right) \geq 3$.

Finally assume $k=2$. Since $\overline{2 i_{\alpha_{0}}} \neq 0$, we have $r_{\alpha_{0}} \geq 3$. Hence $q_{1} q_{2} q_{3} D^{2} \geq$ $3\left(\left(r_{\alpha_{0}}-1\right) / r_{\alpha_{0}}\right) \geq 2$. Further the equality $q_{1} q_{2} q_{3} D^{2}=2$ implies $r_{\alpha_{0}}=3$, $i_{\alpha_{0}}=1$ and $\overline{2 i_{\alpha}}=0$ for $\alpha \neq \alpha_{0}$. Since $\left(i_{\alpha}, r_{\alpha}\right)=1$, we have $\left(r_{\alpha}, i_{\alpha}\right)=(2,1)$ for $\alpha \neq \alpha_{0}$. Hence $N=\sum_{\alpha=1}^{N} i_{\alpha}=6+b_{2}-4 b_{1}=8$ and we obtain the assertions.

(3.6) In the case $b_{1} \geq 3$. We have $q_{1}=q_{2}=q_{3}=1$. By (3.3) for $b_{1}$, we have $D^{2} \geq 2+\sum_{\alpha=1}^{N}\left(\left(i_{\alpha}\left(r_{\alpha}-i_{\alpha}\right)\right) / r_{\alpha}\right) \geq 2$ (cf. the proof of (3.5)). Hence $q_{1} q_{2} q_{3} D^{2} \geq 2$, and the equality holds if and only if $N=0$ and $D^{2}=2$.

(3.7) In the case $b_{1}=2$. We have $q_{1}=q_{2}=1, q_{3} \geq 2$. Let $R_{1}=\mathbf{C} x_{1}+\mathbf{C} x_{2}$, then $x_{1}, x_{2}$ are algebraically independent over $R_{0}$ by Lemma 2.3. Hence $b_{k} \geq$ $k+1$.

First assume $b_{2} \geq 4$. Then we have $q_{3}=2$. By (3.3) for $b_{2}$, we obtain the relation $q_{1} q_{2} q_{3} D^{2}=2 D^{2} \geq 2+\sum_{\alpha=1}^{N}\left(\left(2 i_{\alpha}\left(r_{\alpha}-2 i_{\alpha}\right)\right) / 2 r_{\alpha}\right) \geq 2$. Here the equality $2 D^{2}=2$ holds if and only if $b_{2}=4$ and $r_{\alpha}=2$ for all $\alpha$. Then $i_{\alpha}=1$ for all $\alpha$, and we obtain $N=2$ by (3.4.2).

Next assume $b_{2}=3$. Then we have $q_{3} \geq 3$. By (3.4.2), we obtain $\sum_{\alpha=1}^{N} i_{\alpha}=1$. Hence $N=1$ and $i_{1}=1$. By (3.4), we obtain $D^{2}=\left(r_{1}-1\right) / r_{1}$ and $b_{3}=5-\left(3-r_{1}\right)_{+}$. If $r_{1} \geq 3$, then $b_{3}=5$ and $q_{3}=3$. We obtain $q_{1} q_{2} q_{3} D^{2}=3\left(\left(r_{1}-1\right) / r_{1}\right) \geq 2$. The equality holds if and only if $r_{1}=3$. Finally assume $r_{1}=2$. Then $b_{3}=4, b_{4}=6$ and $q_{3}=4$. In this case we have the relation $q_{1} q_{2} q_{3} D^{2}=2$.

In the rest of this section, we shall study the case that there is $k \geq 2$ where $b_{l}=0$ for $0<l<k-1, b_{k-1}=1$, and $b_{k} \geq 2$.

We begin with the following:

Lemma 3.8. Let $k \geq 3$. Assume that $b_{l}=0$ for $1 \leq l \leq k-2, b_{k-1}=1$ and that $b_{k} \geq 2$. Then $q_{1} q_{2} q_{3} D^{2}>2$.

Proof. We have $q_{1}=k-1, q_{2}=q_{3}=k$. By (3.3) we obtain the following $k^{2} D^{2}=2\left(b_{k}-2\right)+\sum_{\alpha}\left(\left(\overline{k i_{\alpha}}\left(r_{\alpha}-\overline{k i_{\alpha}}\right)\right) / r_{\alpha}\right)$. If $b_{k} \geq 3$, we obtain the relations $q_{1} q_{2} q_{3} D^{2}=(k-1) k^{2} D^{2} \geq(k-1) 2 \geq 4$.

Now assume $b_{k}=2$. We have $0<k^{2} D^{2}=\sum_{\alpha}\left(\left(\overline{k i_{\alpha}}\left(r_{\alpha}-\overline{k i_{\alpha}}\right)\right) / r_{\alpha}\right)$. There is an index $\alpha_{0}$ such that $\overline{k i_{\alpha_{0}}} \neq 0$. We have the relation $q_{1} q_{2} q_{3} D^{2}=$ $(k-1) k^{2} D^{2} \geq(k-1)\left(\left(r_{\alpha_{0}}-1\right) / r_{\alpha_{0}}\right) \geq((k-1) / 2)$. 
Hence if $k \geq 6$, we obtain $q_{1} q_{2} q_{3} D^{2} \geq(5 / 2)>2$.

Next assume $k=5$. We will show the following.

Claim 3.8.1. $\quad 5^{2} D^{2}=\sum_{\alpha}\left(\left(\overline{5 i_{\alpha}}\left(r_{\alpha}-\overline{5 i_{\alpha}}\right)\right) / r_{\alpha}\right)>(1 / 2)$.

Proof of 3.8.1. If not, we have $\sum_{\alpha}\left(\left(\overline{5 i_{\alpha}}\left(r_{\alpha}-\overline{5 i_{\alpha}}\right)\right) / r_{\alpha}\right)=(1 / 2)$. Then $\left(r_{\alpha_{0}}, i_{\alpha_{0}}\right)=(2,1)$ and $\overline{5 i_{\alpha}}=0$ for $\alpha \neq \alpha_{0}$. Hence $r_{\alpha}=5$ for $\alpha \neq \alpha_{0}$. On the other hand, we have the relation $b_{5}=12-\sum_{\alpha}\left(5 i_{\alpha}-r_{\alpha}\right)_{+}-\sum_{\alpha}\left(5 i_{\alpha}-2 r_{\alpha}\right)_{+}$. Then we can easily show $b_{5} \equiv 3(\bmod 5)$. This contradicts to our assumption $b_{5}=2$.

Next we assume $k=4$. We will show the following.

Claim 3.8.2. $\quad 4^{2} D^{2}=\sum_{\alpha}\left(\left(\overline{4 i_{\alpha}}\left(r_{\alpha}-\overline{4 i_{\alpha}}\right)\right) / r_{\alpha}\right)>(2 / 3)$.

Proof of 3.8.2. If $\overline{4 i_{\alpha}} \neq 0$, then $r_{\alpha}=3$ or $r_{\alpha} \geq 5$. Then we have $\left(r_{\alpha}-1\right) / r_{\alpha}=(2 / 3)$ or $\geq 4 / 5$ respectively. Hence we have $\sum_{\alpha}\left(\left(\overline{4 i_{\alpha}}\left(r_{\alpha}-\right.\right.\right.$ $\left.\left.\left.\overline{4 i_{\alpha}}\right)\right) / r_{\alpha}\right) \geq(2 / 3)$. Suppose the equality holds here. Then there exists $\alpha_{0}$ where $\left(r_{\alpha_{0}}, i_{\alpha_{0}}\right)=(3,1)$ and $\overline{4 i_{\alpha}}=0$ for $\alpha \neq \alpha_{0}$. Then $\left(r_{\alpha}, i_{\alpha}\right)=(4,1)$ or $(2,1)$. On the other hand, we have the relation $b_{4}=6-\sum_{\alpha}\left(4 i_{\alpha}-r_{\alpha}\right)_{+}$. Then we can easily show $b_{4}$ is odd. This contradicts to our assumption $b_{4}=2$.

Finally we assume $k=3$. We will show the following.

Claim 3.8.3. $\quad 3^{2} D^{2}=\sum_{\alpha}\left(\left(\overline{3 i_{\alpha}}\left(r_{\alpha}-\overline{3 i_{\alpha}}\right)\right) / r_{\alpha}\right)>1$.

Proof of 3.8.3. Since $b_{1}=0, b_{2}=1$, we have $b_{3}=5-\sum_{\alpha}\left(3 i_{\alpha}-r_{\alpha}\right)_{+}$. Hence $\sum_{\alpha}\left(3 i_{\alpha}-r_{\alpha}\right)_{+}=3$. Remark that $2 i_{\alpha} \leq r_{\alpha}$ and $3 i_{\alpha}<2 r_{\alpha}$ by assumption. So if $\left(3 i_{\alpha}-r_{\alpha}\right)_{+} \neq 0$, we have $\overline{3 i_{\alpha}}=\left(3 i_{\alpha}-r_{\alpha}\right)=\left(3 i_{\alpha}-r_{\alpha}\right)_{+}$, and $2 \cdot \overline{3 i_{\alpha}} \leq r_{\alpha}$.

If there is $\alpha_{0}$ with $\left(3 i_{\alpha_{0}}-r_{\alpha_{0}}\right)_{+}=3$, then $3 \mid r_{\alpha_{0}}$ and we can see $r_{\alpha_{0}} \geq 6$. Hence $\left(\left(\overline{3 i_{\alpha_{0}}}\left(r_{\alpha_{0}}-\overline{3 i_{\alpha_{0}}}\right)\right) / r_{\alpha_{0}}\right) \geq(3 / 2)>1$.

If there are $\alpha_{1}$ and $\alpha_{2}$ with $\left(3 i_{\alpha_{1}}-r_{\alpha_{1}}\right)_{+}=2$ and $\left(3 i_{\alpha_{2}}-r_{\alpha_{2}}\right)_{+}=1$, then we have $r_{\alpha_{1}} \geq 4$ and $r_{\alpha_{2}} \geq 2$. Hence we have $\sum_{\alpha}\left(\left(\overline{3 i_{\alpha}}\left(r_{\alpha}-\overline{3 i_{\alpha}}\right)\right) / r_{\alpha}\right) \geq$ $(3 / 4)+(1 / 2)>1$.

If there $\alpha$ 's with $\left(3 i_{\alpha}-r_{\alpha}\right)_{+} \neq 0$, then we can see $\sum_{\alpha}\left(\left(\overline{3 i_{\alpha}}\left(r_{\alpha}-\overline{3 i_{\alpha}}\right)\right) / r_{\alpha}\right) \geq$ $3 \cdot(1 / 2)>1$.

Now we will assume $k=2$.

(3.9) In the case $b_{1}=1, b_{2} \geq 3$. We have $q_{1}=1, q_{2}=q_{3}=2$. By (3.3) for $b_{2}$, we have $2 D^{2} \geq 1+\sum_{\alpha=1}^{N}\left(\left(2 i_{\alpha}\left(r_{\alpha}-2 i_{\alpha}\right)\right) / 2 r_{\alpha}\right) \geq 1$. Here the equality $q_{1} q_{2} q_{3} D^{2}=4 D^{2}=2$ holds when $b_{2}=3, r_{\alpha}=2$ for all $\alpha$. Then we have $i_{\alpha}=1$ for all $\alpha$ and $N=5$ follows by (3.4.2). 
(3.10) In the rest of this section, we assume that $b_{1}=1, b_{2}=2$. We have $q_{1}=1, q_{2}=2, q_{3} \geq 3$ the relations $\sum_{\alpha=1}^{N} i_{\alpha}=4$, and $\overline{D^{2}=2-\sum_{\alpha=1}}\left(\left(i_{\alpha}\right)^{2} / r_{\alpha}\right)$. We have the formulas: $b_{3}=5-\sum_{\alpha}\left(3 i_{\alpha}-r_{\alpha}\right)_{+}, b_{4}=10-\sum_{\alpha}\left(4 i_{\alpha}-r_{\alpha}\right)_{+}$. By the algebraic independence of two homogeneous elements (2.3), we have $b_{3} \geq 2, b_{4} \geq 3$. In the below we separate the arguments into cases depending on the type $\left(i_{1}, \ldots, i_{N}\right)$. Here we remark that if $D^{2}>1 / 3$, then $q_{1} q_{2} q_{3} D^{2}>2$ by assumptions.

Case 3.10.1. $\quad N=4,\left(i_{1}, i_{2}, i_{3}, i_{4}\right)=(1,1,1,1)$, with $2 \leq r_{1} \leq r_{2} \leq r_{3} \leq$ $r_{4}$.

We have $b_{3}=5-\sum_{\alpha=1}^{4}\left(3-r_{\alpha}\right)_{+}$.

(3.10.1.1) If $b_{3} \geq 3$, then $r_{3} \geq 3$ and $q_{3}=3$. Hence $D^{2}=2-\left(1 / r_{1}\right)-\left(1 / r_{2}\right)-$ $\left(1 / r_{3}\right)-\left(1 / r_{4}\right) \geq 2-(1 / 2) \times 2-(1 / 3) \times 2=1 / 3$ and we obtain $q_{1} q_{2} q_{3} D^{2} \geq 2$. The equality holds if and only if $r_{1}=r_{2}=2, r_{3}=r_{4}=3$.

(3.10.1.2) Next assume $b_{3}=2$. Then $r_{1}=r_{2}=r_{3}=2$ and $r_{4} \geq 3$. Here we have $b_{4}=4-\left(4-r_{4}\right)_{+}$.

(3.10.1.2.1) If $b_{3}=2, b_{4}=4$, then $r_{4} \geq 4$ and $q_{3}=4$. Hence we obtain $D^{2}=(1 / 2)-\left(1 / r_{4}\right) \geq 1 / 4$ and $q_{1} q_{2} q_{3} D^{2} \geq 2$. Here the equality holds if and only if $r_{4}=4$.

(3.10.1.2.2) If $b_{3}=2, b_{4}=3$ then $r_{4}=3$ and $D^{2}=1 / 6$. Now a direct computation shows $q_{3}=6$, hence $q_{1} q_{2} q_{3} D^{2}=2$ holds in this case.

Case 3.10.2. $\quad N=3,\left(i_{1}, i_{2}, i_{3}\right)=(2,1,1)$ with $r_{1} \geq 5,\left(r_{1}, 2\right)=1$, and $2 \leq r_{2} \leq r_{3}$

We have $b_{3}=5-\left(6-r_{1}\right)_{+}-\left(3-r_{2}\right)_{+}-\left(3-r_{3}\right)_{+}$.

(3.10.2.1) If $b_{3} \geq 3$, then we have $r_{1} \geq 7$ or $r_{3} \geq 3$. In these two cases, we can easily show $D^{2}>1 / 3$ and $q_{1} q_{2} q_{3} D^{2}>2$.

(3.10.2.2) If $b_{3}=2$, then $r_{1}=5, r_{2}=r_{3}=2$, and $D^{2}=1 / 5$. Now a direct computation shows $q_{3}=5$, hence $q_{1} q_{2} q_{3} D^{2}=2$ holds.

Case 3.10.3. $\quad N=2,\left(i_{1}, i_{2}\right)=(3,1)$, with $r_{1} \geq 7,\left(r_{1}, 3\right)=1, r_{2} \geq 2$.

We have $b_{3}=5-\left(9-r_{1}\right)_{+}-\left(3-r_{2}\right)_{+}$.

(3.10.3.1) If $b_{3} \geq 3$, then $r_{1} \geq 8$ or $r_{2} \geq 3$. In these two cases, we can easily show $D^{2}>1 / 3$ and $q_{1} q_{2} q_{3} D^{2}>2$.

(3.10.3.2) If $b_{3}=2$, then $r_{1}=7, r_{2}=2$ and $D^{2}=3 / 14$. Now a direct computation shows $q_{3}=5$, hence $q_{1} q_{2} q_{3} D^{2}>2$.

Case 3.10.4. $\quad N=2,\left(i_{1}, i_{2}\right)=(2,2)$, with $5 \leq r_{1} \leq r_{2},\left(r_{i}, 2\right)=1, i=$ 1,2 . 
We have $D^{2} \geq 2-(4 / 5) \times 2=(2 / 5)>(1 / 3)$, hence $q_{1} q_{2} q_{3} D^{2}>2$.

Case 3.10.5. $\quad N=1, i_{1}=4, r_{1} \geq 9,\left(r_{1}, 4\right)=1$.

We have $b_{3}=5-\left(12-r_{1}\right)_{+}$.

(3.10.5.1) If $b_{3} \geq 3$, then $r_{1} \geq 10$ and $D^{2} \geq 2-(16 / 11)=(6 / 11)>(1 / 3)$. Hence $q_{1} q_{2} q_{3} D^{2}>2$.

(3.10.5.2) If $b_{3}=2$, then $r_{1}=9$ and $D^{2}=2 / 9$. Now a direct computation shows $q_{3}=5$, hence $q_{1} q_{2} q_{3} D^{2}>2$.

\section{$\S 4$. The Case $b_{1}=b_{2}=0$ and $b_{3} \leq 1, b_{4} \leq 1$}

(4.1) In this section, we assume that $b_{1}=0, b_{2}=0, b_{3} \leq 1, b_{4} \leq 1$. By (3.4) we have $\sum_{\alpha=1}^{N} i_{\alpha}=6$. Hence we have the following basic cases for $\mathcal{I}=\left(i_{1}, \ldots, i_{N}\right)$ and $r_{i}$ 's:

I. $N=6, \mathcal{I}=(1,1,1,1,1,1)$ with $2 \leq r_{1} \leq \cdots \leq r_{6}$.

II. $N=5, \mathcal{I}=(2,1,1,1,1)$ with $r_{1} \geq 5,\left(r_{1}, 2\right)=1,2 \leq r_{2} \leq \cdots \leq r_{5}$.

III. $N=4, \mathcal{I}=(3,1,1,1)$ with $r_{1} \geq 7,\left(r_{1}, 3\right)=1,2 \leq r_{2} \leq \cdots \leq r_{4}$.

IV. $N=4, \mathcal{I}=(2,2,1,1)$ with $5 \leq r_{1} \leq r_{2},\left(r_{1}, 2\right)=\left(r_{2}, 2\right)=1,2 \leq r_{3} \leq r_{4}$.

V. $N=3, \mathcal{I}=(4,1,1)$ with $r_{1} \geq 9,\left(r_{1}, 4\right)=1,2 \leq r_{2} \leq r_{3}$.

VI. $N=3, \mathcal{I}=(3,2,1)$ with $r_{1} \geq 7,\left(r_{1}, 3\right)=1, r_{2} \geq 5,\left(r_{2}, 2\right)=1,2 \leq r_{3}$.

VII. $N=3, \mathcal{I}=(2,2,2)$ with $5 \leq r_{1} \leq r_{2} \leq r_{3},\left(r_{i}, 2\right)=1$ for $i=1,2,3$.

VIII. $N=2, \mathcal{I}=(5,1)$ with $r_{1} \geq 11,\left(r_{1}, 5\right)=1, r_{2} \geq 2$.

IX. $N=2, \mathcal{I}=(4,2)$ with $r_{1} \geq 9,\left(r_{1}, 4\right)=1, r_{2} \geq 5,\left(r_{2}, 2\right)=1$.

X. $N=2, \mathcal{I}=(3,3)$ with $7 \leq r_{1} \leq r_{2},\left(r_{i}, 3\right)=1$ for $i=1,2$.

XI. $N=1, \mathcal{I}=(6)$ with $r_{1} \geq 13,\left(r_{1}, 6\right)=1$.

In these cases, we can show $q_{1} q_{2} q_{3} D^{2} \geq 2$ for I-VI and $q_{1} q_{2} q_{3} D^{2}>2$ for VII-XI. In the below we will discuss the cases I-VI according to the types of $b_{3}, b_{4}$. We have the relations (3.4.3): $b_{3}=2-\sum_{\alpha}\left(3 i_{\alpha}-r_{\alpha}\right)_{+}, b_{4}=6-$ $\sum_{\alpha}\left(4 i_{\alpha}-r_{\alpha}\right)_{+}$. By using these we can show the following. 
Lemma 4.2. Assume $b_{3}=b_{4}=1$. Then, for the cases I-VI, one of the followings occurs.

$$
\begin{gathered}
I_{1,1} 1: r_{1}=r_{2}=r_{3}=r_{4}=3,4 \leq r_{5} \leq r_{6} . \\
I I_{1,1} 1: r_{1}=5, r_{2}=r_{3}=3,4 \leq r_{4} \leq r_{5}, I I_{1,1} 2: r_{1}=7, r_{2}=2, r_{3}=r_{4}= \\
3, r_{5} \geq 4, I I_{1,1} 3: r_{1} \geq 9, r_{2}=2, r_{3}=r_{4}=r_{5}=3 .
\end{gathered}
$$$$
I I I_{1,1} 1: r_{1}=8, r_{2}=3,4 \leq r_{3} \leq r_{4}, I I I_{1,1} 2: r_{1}=10, r_{2}=2, r_{3}=3, r_{4} \geq 4,
$$
$I I I_{1,1} 3: r_{1}=11, r_{2}=2, r_{3}=r_{4}=3$.

$I V_{1,1} 1: r_{1}=5, r_{2}=7, r_{3}=3,4 \leq r_{4}, I V_{1,1} 2: r_{1}=5, r_{2} \geq 9, r_{3}=r_{4}=3$, $I V_{1,1} 3: r_{1}=r_{2}=7, r_{3}=2, r_{4}=3$.

$V_{1,1} 1: r_{1}=11,4 \leq r_{2} \leq r_{3}, V_{1,1} 2: r_{1}=13, r_{2}=2, r_{3} \geq 4$.

$V I_{1,1} 1: r_{1}=8, r_{2}=7, r_{3} \geq 4, V I_{1,1} 2: r_{1}=8, r_{2} \geq 9, r_{3}=3, V I_{1,1} 3: r_{1}=$ $10, r_{2}=5, r_{3} \geq 4, V I_{1,1} 4: r_{1}=11, r_{2}=5, r_{3}=3, V I_{1,1} 5: r_{1}=10, r_{2}=7, r_{3}=$ 2 .

(4.3) Proof of the Theorem $\mathrm{C}$ for the cases of 4.2. Here we have $q_{1}=3, q_{2}=$ $4, q_{3} \geq 5$, and $D^{2}=2-\sum_{\alpha}\left(\left(i_{\alpha}\right)^{2} / r_{\alpha}\right)$. We can easily see $D^{2}>(1 / 30)$ for $\mathrm{I}_{1,1} 1, \mathrm{II}_{1,1} 3, \mathrm{III}_{1,1} 1, \mathrm{IV}_{1,1} 1, \mathrm{IV}_{1,1} 2, \mathrm{~V}_{1,1} 1, \mathrm{VI}_{1,1} 1, \mathrm{VI}_{1,1} 2, \mathrm{VI}_{1,1} 3, \mathrm{VI}_{1,1} 4$. Hence $q_{1} q_{2} q_{3} D^{2}>2$ holds for these cases.

$\left(\mathrm{II}_{1,1} 1\right)$ We have $D^{2}=(8 / 15)-\left(1 / r_{4}\right)-\left(1 / r_{5}\right) \geq 1 / 30$. Hence $q_{1} q_{2} q_{3} D^{2} \geq 2$. Here the equality holds if and only if $r_{4}=r_{5}=4$.

$\left(\mathrm{II}_{1,1} 2\right)$ We have $D^{2}=(11 / 42)-\left(1 / r_{5}\right)$. If $r_{5} \geq 5$, then $D^{2}>(1 / 20)$ and $q_{1} q_{2} q_{3} D^{2}>2$. Now assume $r_{5}=4$. Then $D^{2}=1 / 84$. A computation shows $q_{3}=14$. Hence $q_{1} q_{2} q_{3} D^{2}=2$ follows.

$\left(\mathrm{III}_{1,1} 2\right)$ We have $D^{2}=(4 / 15)-\left(1 / r_{4}\right)$. If $r_{4} \geq 5$, then $D^{2}>(1 / 30)$ and $q_{1} q_{2} q_{3} D^{2}>2$. Now assume $r_{4}=4$. Then $D^{2}=1 / 60$. A computation shows $q_{3}=10$. Hence $q_{1} q_{2} q_{3} D^{2}=2$ follows.

$\left(\mathrm{III}_{1,1} 3\right)$ We have $D^{2}=1 / 66$. A computation shows $q_{3}=11$. Hence $q_{1} q_{2} q_{3} D^{2}$ $=2$ follows.

$\left(\mathrm{IV}_{1,1} 3\right)$ We have $D^{2}=1 / 42$. A computation shows $q_{3}=7$. Hence $q_{1} q_{2} q_{3} D^{2}=$ 2 follows.

$\left(\mathrm{V}_{1,1} 2\right)$ We have $D^{2}=(7 / 26)-\left(1 / r_{3}\right)$. If $r_{3} \geq 5$, then $D^{2}>(1 / 30)$ and $q_{1} q_{2} q_{3} D^{2}>2$. Now assume $r_{3}=4$. Then $D^{2}=1 / 52$. A computation shows $q_{3}=10$. Hence $q_{1} q_{2} q_{3} D^{2}>2$.

$\left(\mathrm{VI}_{1,1} 5\right)$ We have $D^{2}=1 / 35$. A computation shows $q_{3}=7$. Hence $q_{1} q_{2} q_{3} D^{2}>$ 2 .

Lemma 4.4. Assume $b_{3}=1, b_{4}=0$. Then, for the cases I-VI, one of the followings occurs.

$$
I_{1,0} 1: r_{1}=2, r_{2}=r_{3}=r_{4}=r_{5}=3, r_{6} \geq 4 \text {. }
$$




$$
\begin{aligned}
& I I_{1,0} 1: r_{1}=5, r_{2}=r_{3}=r_{4}=3, r_{5} \geq 4, I I_{1,0} 2: r_{1}=7, r_{2}=2, r_{3}=r_{4}= \\
r_{5}= & 3 . \\
& I I I_{1,0} 1: r_{1}=8, r_{2}=r_{3}=3, r_{4} \geq 4, I I I_{1,0} 2: r_{1}=10, r_{2}=2, r_{3}=r_{4}=3 . \\
& I V_{1,0} 1: r_{1}=5, r_{2}=7, r_{3}=r_{4}=3 . \\
& V_{1,0} 1: r_{1}=11, r_{2}=3, r_{4} \geq 4, V_{1,0} 2: r_{1}=13, r_{2}=2, r_{3}=3 . \\
& V I_{1,0} 1: r_{1}=8, r_{2}=7, r_{3}=3, V I_{1,0} 2: r_{1}=10, r_{2}=5, r_{3}=3 .
\end{aligned}
$$

(4.5) Proof of the Theorem $\mathrm{C}$ for the cases of 4.4. Here we have $q_{1}=3, q_{3} \geq$ $q_{2} \geq 5$, and $D^{2}=2-\sum_{\alpha}\left(\left(i_{\alpha}\right)^{2} / r_{\alpha}\right)$. We can easily see $D^{2}<0$ for $\mathrm{II}_{1,0} 2$, $\mathrm{III}_{1,0} 2, \mathrm{IV}_{1,0} 1, \mathrm{~V}_{1,0} 2, \mathrm{VI}_{1,0} 1, \mathrm{VI}_{1,0} 2$. Hence these cases do not happen.

$\left(\mathrm{I}_{1,0} 1\right)$ Since $0<D^{2}=(1 / 6)-\left(1 / r_{6}\right)$, we have $r_{6} \geq 7$. If $r_{6} \geq 8$, then $D^{2} \geq 1 / 24$ and $q_{1} q_{2} q_{3} D^{2}>2$. Now assume $r_{6}=7$. Then $D^{2}=1 / 42$. A direct computation shows $\left(q_{1}, q_{2}, q_{3}\right)=(3,6,7)$. Hence $q_{1} q_{2} q_{3} D^{2}=3$.

$\left(\mathrm{II}_{1,0} 1\right)$ Since $0<D^{2}=(1 / 5)-\left(1 / r_{5}\right)$, we have $r_{5} \geq 6$. Hence $D^{2} \geq(1 / 30)$ and $q_{1} q_{2} q_{3} D^{2}>2$.

(III 1,01$)$ Since $0<D^{2}=(5 / 24)-\left(1 / r_{4}\right)$, we have $r_{4} \geq 5$. If $r_{4} \geq 6$, then $D^{2} \geq 1 / 24$. Hence $q_{1} q_{2} q_{3} D^{2}>2$. Now assume $r_{4}=5$. Then $D^{2}=1 / 120$. Here we can show $\left(q_{1}, q_{2}, q_{3}\right)=(3,5,16)$. Hence we obtain $q_{1} q_{2} q_{3} D^{2}=2$.

$\left(\mathrm{V}_{1,0} 1\right)$ Since $0<D^{2}=(7 / 33)-\left(1 / r_{3}\right)$, we have $r_{3} \geq 5$. If $r_{3} \geq 6$, then $D^{2}>(1 / 30)$ and $q_{1} q_{2} q_{3} D^{2}>2$. Now assume $r_{3}=5$. Then $D^{2}=2 / 165$. Here we can show $\left(q_{1}, q_{2}, q_{3}\right)=(3,5,11)$. Hence we obtain $q_{1} q_{2} q_{3} D^{2}=2$.

Lemma 4.6. Assume $b_{3}=0, b_{4}=1, b_{5} \leq 1$. Then, for the cases $\mathrm{I}-\mathrm{VI}$, one of the followings occurs.

$I_{0,1} 1: r_{1}=r_{2}=2, r_{3}=3, r_{4}=4 \leq r_{5} \leq r_{6}$.

$I I_{0,1} 1: r_{1}=5, r_{2}=2, r_{3}=r_{4}=4 \leq r_{5}, I I_{0,1} 2: r_{1}=7, r_{2}=r_{3}=2, r_{4} \geq$ $4, r_{5} \geq 5, I I_{0,1} 3: r_{1}=9, r_{2}=r_{3}=2, r_{4}=3, r_{5} \geq 5, I I_{0,1} 4: r_{1} \geq 11, r_{2}=r_{3}=$ $2, r_{4}=3, r_{5}=4$.

$I I I_{0,1} 1: r_{1}=7, r_{2}=r_{3}=4, r_{4} \geq 4, I I I_{0,1} 2: r_{1}=13, r_{2}=r_{3}=2, r_{4}=3$, $I I I_{0,1} 3: r_{1}=14, r_{2}=r_{3}=2, r_{4}=3, I I I_{0,1} 4: r_{1}=11, r_{2}=r_{3}=2, r_{4} \geq 4$.

$I V_{0,1} 1: r_{1}=5, r_{2}=9, r_{3}=2, r_{4}=4, I V_{0,1} 2: r_{1}=7, r_{2} \geq 9, r_{3}=r_{4}=2$.

$V_{0,1} 1: r_{1}=15, r_{2}=r_{3}=2$.

$V I_{0,1} 1: r_{1}=7, r_{2}=9, r_{3}=4, V I_{0,1} 2: r_{1} \geq 10, r_{2}=5, r_{3}=2$.

(4.7) Proof of the Theorem $\mathrm{C}$ for the cases of 4.6. Here we have $q_{1}=4, q_{2} \geq$ $5, q_{3} \geq 6$, and $D^{2}=2-\sum_{\alpha}\left(\left(i_{\alpha}\right)^{2} / r_{\alpha}\right)$. We can easily see $D^{2}>(1 / 60)$ for $\mathrm{II}_{1,0} 3, \mathrm{II}_{1,0} 4, \mathrm{III}_{0,1} 3$. Hence $q_{1} q_{2} q_{3} D^{2}>2$ holds for these cases. Further we can see $D^{2}<0$ for III $_{0,1} 2$ and $\mathrm{V}_{0,1} 2$. Hence these cases do not happen.

$\left(\mathrm{I}_{0,1} 1\right)$ We have $b_{5}=1-\left(5-r_{5}\right)_{+}-\left(5-r_{6}\right)_{+}$. 
If $b_{5}=1$, then $5 \leq r_{4} \leq r_{5}$. Hence $D^{2}=(5 / 12)-\left(1 / r_{5}\right)-\left(1 / r_{6}\right) \geq 1 / 60$. Hence $q_{1} q_{2} q_{3} D^{2} \geq 2$. Here the equality holds if and only if $r_{5}=r_{6}=5$.

If $b_{5}=0$, then $r_{5}=4, r_{6} \geq 5$. Since $0<D^{2}=(1 / 6)-\left(1 / r_{6}\right)$, we have $r_{6} \geq 7$. Hence $D^{2}>(1 / 60)$, and $q_{1} q_{2} q_{3} D^{2}>2$.

$\left(\mathrm{II}_{0,1} 1\right)$ Since $0<D^{2}=(1 / 5)-\left(1 / r_{5}\right)$, we have $r_{5} \geq 6$. Hence then $D^{2}>(1 / 60)$ and $q_{1} q_{2} q_{3} D^{2}>2$.

$\left(\mathrm{II}_{0,1} 2\right)$ We have $b_{5}=1-\left(5-r_{4}\right)_{+}-\left(5-r_{5}\right)_{+}$.

If $b_{5}=1$, then $5 \leq r_{4} \leq r_{5}$. Hence $D^{2}=(3 / 7)-\left(1 / r_{4}\right)-\left(1 / r_{5}\right)>1 / 60$. Hence $q_{1} q_{2} q_{3} D^{2}>2$.

If $b_{5}=0$, then $r_{4}=4, r_{5} \geq 5$. Since $0<D^{2}=(5 / 28)-\left(1 / r_{5}\right)$, we have $r_{5} \geq 6$. Here $D^{2} \geq(1 / 84)$. Further we can see $b_{6}=1$ and $q_{2}=6, q_{3} \geq 7$. Hence $q_{1} q_{2} q_{3} D^{2} \geq 2$ follows. We can check the equality holds when $r_{5}=6$.

$\left(\mathrm{III}_{0,1} 1\right)$ Since $0<D^{2}=(3 / 14)-\left(1 / r_{4}\right)$, we have $r_{4} \geq 5$. If $r_{4} \geq 6$, we have $D^{2}>(1 / 60)$ and $q_{1} q_{2} q_{3} D^{2}>2$. Now assume $r_{4}=5$. Then we can see $\left(q_{1}, q_{2}, q_{3}\right)=(4,5,7)$. Hence $q_{1} q_{2} q_{3} D^{2}=2$ follows.

$\left(\mathrm{III}_{0,1} 4\right)$ Since $0<D^{2}=(2 / 11)-\left(1 / r_{4}\right)$, we have $r_{4} \geq 6$. Here we have $D^{2} \geq(1 / 66)$. We can see $b_{5}=0$ and $q_{2} \geq 6$. Hence $q_{1} q_{2} q_{3} D^{2}>2$ follows.

$\left(\mathrm{IV}_{0,1} 1\right)$ We can check $D^{2}=1 / 180$ and $\left(q_{1}, q_{2}, q_{3}\right)=(4,5,18)$. Hence $q_{1} q_{2} q_{3} D^{2}$ $=2$ holds.

$\left(\mathrm{IV}_{0,1} 2\right)$ Since $0<D^{2}=(3 / 7)-\left(4 / r_{2}\right)$, we have $r_{2} \geq 11$. Here $D^{2}>(1 / 60)$ and $q_{1} q_{2} q_{3} D^{2}>2$.

$\left(\mathrm{VI}_{0,1} 1\right)$ We can check $D^{2}=5 / 252$ and $\left(q_{1}, q_{2}, q_{3}\right)=(4,5,7)$. Hence $q_{1} q_{2} q_{3} D^{2}$ $>2$ holds.

$\left(\mathrm{VI}_{0,1} 2\right)$ Since $0<D^{2}=(7 / 10)-\left(9 / r_{1}\right)$, we have $r_{1} \geq 13$ and $D^{2} \geq(1 / 130)$. If $r_{1} \geq 14$, then $D^{2} \geq(4 / 70)>(1 / 30)$. Hence $q_{1} q_{2} q_{3} D^{2}>2$. Now assume $r_{1}=13$. Here we can show $\left(q_{1}, q_{2}, q_{3}\right)=(4,5,13)$ and $q_{1} q_{2} q_{3} D^{2}=2$ follows.

Lemma 4.8. Assume $b_{3}=b_{4}=0, b_{5} \leq 1, b_{6} \leq 1$. Then, for the cases I-VI, one of the followings occurs.

$I_{0,0} 1: r_{1}=r_{2}=2, r_{3}=r_{4}=3,4 \leq r_{5} \leq r_{6}$.

$I I_{0,0} 1: r_{1}=5, r_{2}=2, r_{3}=3,4 \leq r_{4} \leq r_{5}, I I_{0,0} 2: r_{1} \geq 9, r_{2}=r_{3}=2, r_{4}=$ $r_{5}=3, I I_{0,0} 3: r_{1}=7, r_{2}=r_{3}=2, r_{4}=3, r_{5} \geq 4$.

$I I I_{0,0} 1: r_{1}=7, r_{2}=3,4 \leq r_{3} \leq r_{4}, I I I_{0,0} 2: r_{1}=8, r_{2}=2,4 \leq r_{3} \leq r_{4}$, $I I I_{0,0} 3: r_{1}=10, r_{2}=r_{3}=2, r_{4} \geq 4, I I I_{0,0} 4: r_{1}=11, r_{2}=r_{3}=2, r_{4}=3$.

$I V_{0,0} 1: r_{1}=r_{2}=5,4 \leq r_{3} \leq r_{4}, I V_{0,0} 2: r_{1}=5, r_{2}=7, r_{3}=2, r_{4} \geq 4$, $I V_{0,0} 3: r_{1}=5, r_{2} \geq 9, r_{3}=2, r_{4}=3 . I V_{0,0} 4: r_{1}=r_{2}=7, r_{3}=r_{4}=2$.

$V_{0,0}:$ There are no such cases.

$V I_{0,0} 1: r_{1}=r_{2}=7, r_{3} \geq 4, V I_{0,0} 2: r_{1}=7, r_{2} \geq 9, r_{3}=3 V I_{0,0} 3: r_{1}=$ $8, r_{2} \geq 7, r_{3}=2$. 
(4.9) Proof of the Theorem $\mathrm{C}$ for the cases of 4.8. Here we have $q_{1} \geq 5, q_{2} \geq$ $6, q_{3} \geq 7$, and $D^{2}=2-\sum_{\alpha}\left(\left(i_{\alpha}\right)^{2} / r_{\alpha}\right)$. We can easily see $D^{2}>(1 / 105)$ for $\mathrm{V}_{0,0} 1$. Hence $q_{1} q_{2} q_{3} D^{2}>2$ holds for these cases. Further we can see $D^{2}<0$ for $\mathrm{III}_{0,0} 4, \mathrm{IV}_{0,0} 4$. Hence these cases do not happen.

$\left(\mathrm{I}_{0,0} 1\right)$ Since $b_{5}=-\left(5-r_{5}\right)_{+}-\left(5-r_{6}\right)_{+} \leq 0$, we have $b_{5}=0$ and $r_{5} \geq 5$. Here $b_{6}=2-\left(6-r_{5}\right)_{+}-\left(6-r_{6}\right)_{+}$. We have assumed that $b_{6} \leq 1$. Hence $r_{5}=5$. Since $0<D^{2}=(2 / 15)-\left(1 / r_{6}\right)$, we have $r_{6} \geq 8$. Now $b_{6}=1$. We obtain $q_{1}=6, q_{2} \geq 7$ and $D^{2} \geq(1 / 120)$. Hence $q_{1} q_{2} q_{3} D^{2}>2$.

$\left(\mathrm{II}_{0,0} 1\right)$ We have $b_{5}=1-\left(5-r_{4}\right)_{+}-\left(5-r_{5}\right)_{+}$.

If $b_{5}=1$, then $r_{4} \geq 5$. Since we have assumed $1 \geq b_{6}=2-\left(6-r_{4}\right)_{+}-(6-$ $\left.r_{5}\right), r_{4}=5$. Since $0<D^{2}=(1 / 6)-\left(1 / r_{5}\right)$, we have $r_{5} \geq 7$ and $D^{2} \geq(1 / 42)$. Hence $q_{1} q_{2} q_{3} D^{2}>2$.

Now assume $b_{5}=0$. We have $r_{4}=4, r_{5} \geq 5$. Since $0<D^{2}=(7 / 60)-$ $\left(1 / r_{5}\right)$, we have $r_{5} \geq 9$ and $D^{2} \geq(1 / 180)$. On the other hand we have $b_{6}=-$ $\left(6-r_{5}\right)_{+}=0$. We may assume $b_{7} \leq 1, b_{8} \leq 1$ by Section 3 . Hence $q_{1} q_{2} q_{3} D^{2}>2$. $\left(\mathrm{II}_{0,0} 2\right)$ Since $0<D^{2}=(1 / 3)-\left(4 / r_{1}\right)$, we have $r_{1} \geq 13$ and $D^{2} \geq(1 / 39)$. Hence $q_{1} q_{2} q_{3} D^{2}>2$.

$\left(\mathrm{II}_{0,0} 3\right)$ We have $b_{5}=-1-\left(5-r_{5}\right)_{+} \leq-1$. Hence this case dose not happen. $\left(\mathrm{III}_{0,1} 1\right)$ We have $b_{5}=1-\left(5-r_{3}\right)_{+}-\left(5-r_{4}\right)_{+}$.

If $b_{5}=1$, then $r_{3} \geq 5$. Since we have assumed $1 \geq b_{6}=2-\left(6-r_{3}\right)_{+}-$ $\left(6-r_{4}\right)_{+}$, we obtain $r_{3}=5$. Since $0<D^{2}=(19 / 105)-\left(1 / r_{4}\right)$, we have $r_{4} \geq 6$ and $D^{2} \geq(1 / 70)$. Therefore $q_{1} q_{2} q_{3} D^{2}>2$.

Now assume $b_{5}=0$. We have $r_{3}=4, r_{4} \geq 5$. Now $b_{6}=-\left(6-r_{4}\right)_{+}$, hence $b_{6}=0$ and $r_{4} \geq 6$. Since $0<D^{2}=(11 / 84)-\left(1 / r_{4}\right)$, we have $r_{4} \geq 8$ and $D^{2} \geq(1 / 168)$. We may assume $b_{7} \leq 1, b_{8} \leq 1$ by Section 3 . Hence $q_{1} q_{2} q_{3} D^{2}>2$.

$\left(\mathrm{III}_{0,0} 2\right)$ We have $b_{5}=1-\left(5-r_{3}\right)_{+}-\left(5-r_{4}\right)_{+}$.

If $b_{5}=1$, then $r_{3} \geq 5$. Since we have assumed $1 \geq b_{6}=2-\left(6-r_{3}\right)_{+}-$ $\left(6-r_{4}\right)_{+}$, we have $r_{3}=5$, and $q_{1}=5, q_{2} \geq 6, q_{3} \geq 7$. Since $0<D^{2}=$ $(7 / 40)-\left(1 / r_{4}\right)$, we have $r_{4} \geq 6$. In the case $r_{4} \geq 7, q_{1} q_{2} q_{3} D^{2}>2$ follows. Now assume $r_{4}=6$. Then we can check $\left(q_{1}, q_{2}, q_{3}\right)=(5,6,8)$ and $D^{2}=1 / 120$. Therefore $q_{1} q_{2} q_{3} D^{2}=2$ follows.

Now assume $b_{5}=0$. Then $r_{3}=4, r_{4} \geq 5$. Since $0<D^{2}=(1 / 8)-\left(1 / r_{4}\right)$, we have $r_{4} \geq 9$ and $D^{2} \geq(1 / 72)$. Hence $q_{1} q_{2} q_{3} D^{2}>2$.

$\left(\mathrm{III}_{0,0} 3\right)$ We have $b_{5}=-1-\left(5-r_{4}\right)_{+} \leq-1$. Hence this case does not happen. $\left(\mathrm{IV}_{0,0} 1\right) \quad$ Since $1 \geq b_{5}=2-\left(5-r_{3}\right)_{+}-\left(5-r_{4}\right)_{+}$, we have $r_{3}=4$. Since $0<D^{2}=(3 / 20)-\left(1 / r_{4}\right)$, we have $r_{4} \geq 7$ and $D^{2} \geq(1 / 140)$. Then we can see $b_{5}=1, b_{6}=0, b_{7}=1$ and $q_{1}=5, q_{2}=7, q_{3} \geq 8$. Hence, $q_{1} q_{2} q_{3} D^{2}>2$ when 
$r_{4} \geq 8$. For $r_{4}=7$, we can see $q_{3}=8$ and $q_{1} q_{2} q_{3} D^{2}=2$.

$\left(\mathrm{IV}_{0,0} 2\right)$ Since $0<D^{2}=(9 / 70)-\left(1 / r_{4}\right)$, we have $r_{4} \geq 8$ and $D^{2} \geq(1 / 280)$. Here we see $b_{5}=0, b_{6}=0, b_{7}=1, b_{8}=1, b_{9}=1-\left(9-r_{4}\right)_{+}$. Hence $q_{1}=7, q_{2}=8, q_{3} \geq 9$. If $r_{4} \geq 9, D^{2} \geq(11 / 630)$ and $q_{1} q_{2} q_{3} D^{2}>2$. Further, if $r_{4}=8$, then we can see $q_{3}=10$ and $q_{1} q_{2} q_{3} D^{2}=2$.

$\left(\mathrm{IV}_{0,0} 3\right)$ Since $0<D^{2}=(11 / 30)-\left(4 / r_{2}\right)$, we have $r_{2} \geq 11$ and $D^{2} \geq(1 / 330)$. If $r_{2} \neq 11$, then $r_{2} \geq 13$ and $D^{2}>(1 / 30)$, hence $q_{1} q_{2} q_{3} D^{2}>2$. In the case $r_{2}=11$, we can see $\left(q_{1}, q_{2}, q_{3}\right)=(5,6,22)$ and $D^{2}=1 / 330$. Hence $q_{1} q_{2} q_{3} D^{2}=2$.

$\left(\mathrm{VI}_{0,0} 1\right)$ Since $0<D^{2}=(1 / 7)-\left(1 / r_{3}\right)$, we have $r_{3} \geq 8$ and $D^{2}>(1 / 105)$. Hence $q_{1} q_{2} q_{3} D^{2}>2$.

$\left(\mathrm{VI}_{0,0} 2\right)$ Since $0<D^{2}=(8 / 21)-\left(4 / r_{2}\right)$, we have $r_{2} \geq 11$ and $D^{2} \geq(1 / 105)$. Hence $q_{1} q_{2} q_{3} D^{2}>2$.

$\left(\mathrm{VI}_{0,0} 3\right)$ Since $D^{2}=(3 / 8)-\left(4 / r_{2}\right)$, we have $r_{2} \geq 11$ and $D^{2} \geq(1 / 88)$. Hence $q_{1} q_{2} q_{3} D^{2}>2$.

(4.10) Now one can show $q_{1} q_{2} q_{3} D^{2}>2$ for the cases VII-XII by similar arguments. These are not difficult. Hence we left the details to the readers and we omit them.

\section{$\S 5$. The Case of $b_{1}=0, b_{2}=1$ and $b_{3} \leq 1$}

(5.1) In this section, we assume that $b_{1}=0, b_{2}=1, b_{3} \leq 1$. By (3.4) we have $\sum_{\alpha=1}^{N} i_{\alpha}=7$. Hence we have the following basic cases for $\mathcal{I}=\left(i_{1}, \ldots, i_{N}\right)$ and $r_{i}$ 's:

I. $N=7, \mathcal{I}=(1,1,1,1,1,1,1)$ with $2 \leq r_{1} \leq \cdots \leq r_{7}$.

II. $N=6, \mathcal{I}=(2,1,1,1,1,1)$ with $r_{1} \geq 5,\left(r_{1}, 2\right)=1,2 \leq r_{2} \leq \cdots \leq r_{6}$.

III. $N=5, \mathcal{I}=(3,1,1,1,1)$ with $r_{1} \geq 7,\left(r_{1}, 3\right)=1,2 \leq r_{2} \leq \cdots \leq r_{5}$.

IV. $N=5, \mathcal{I}=(2,2,1,1,1)$ with $5 \leq r_{1} \leq r_{2},\left(r_{1}, 2\right)=\left(r_{2}, 2\right)=1,2 \leq r_{3} \leq$ $r_{4} \leq r_{5}$.

V. $N=4, \mathcal{I}=(4,1,1,1)$ with $r_{1} \geq 9,\left(r_{1}, 4\right)=1,2 \leq r_{2} \leq r_{3} \leq r_{4}$.

VI. $N=4, \mathcal{I}=(3,2,1,1)$ with $r_{1} \geq 7,\left(r_{1}, 3\right)=1, r_{2} \geq 5,\left(r_{2}, 2\right)=1$, $2 \leq r_{3} \leq r_{4}$.

VII. $N=4, \mathcal{I}=(2,2,2,1)$ with $5 \leq r_{1} \leq r_{2} \leq r_{3},\left(r_{i}, 2\right)=1$ for $i=1,2,3$, $r_{4} \geq 2$.

VIII. $N=3, \mathcal{I}=(5,1,1)$ with $r_{1} \geq 11,\left(r_{1}, 5\right)=1,2 \leq r_{2} \leq r_{3}$. 
IX. $N=3, \mathcal{I}=(4,2,1)$ with $r_{1} \geq 9,\left(r_{1}, 4\right)=1, r_{2} \geq 5,\left(r_{2}, 2\right)=1, r_{3} \geq 2$.

X. $N=3, \mathcal{I}=(3,3,1)$ with $7 \leq r_{1} \leq r_{2},\left(r_{i}, 3\right)=1$ for $i=1,2, r_{3} \geq 2$.

XI. $N=3, \mathcal{I}=(3,2,2)$ with $r_{1} \geq 7,\left(r_{1}, 3\right)=1,5 \leq r_{2} \leq r_{3},\left(r_{2}, 2\right)=$ $\left(r_{3}, 2\right)=1$.

XII. $N=2, \mathcal{I}=(6,1)$ with $r_{1} \geq 13,\left(r_{1}, 6\right)=1, r_{2} \geq 2$.

XIII. $N=2, \mathcal{I}=(5,2)$ with $r_{1} \geq 11,\left(r_{1}, 5\right)=1, r_{2} \geq 5,\left(r_{2}, 2\right)=1$.

XIV. $N=2, \mathcal{I}=(4,3)$ with $r_{1} \geq 9,\left(r_{1}, 4\right)=1, r_{2} \geq 7,\left(r_{2}, 2\right)=1$.

XV. $N=1, \mathcal{I}=(7)$ with $r_{1} \geq 15,\left(r_{1}, 7\right)=1$.

In these cases, we can show $q_{1} q_{2} q_{3} D^{2} \geq 2$ for I-VI and $q_{1} q_{2} q_{3} D^{2}>2$ for VII-XV. In the below we will discuss the cases I-VI according to the types of $b_{3}$. We have the relations (3.4.3): $b_{3}=5-\sum_{\alpha}\left(3 i_{\alpha}-r_{\alpha}\right)_{+}, b_{4}=12-\sum_{\alpha}\left(4 i_{\alpha}-r_{\alpha}\right)_{+}$. By using these we can show the following.

Lemma 5.2. Assume $b_{3}=1$. Then, for the cases I-VI, one of the followings occurs.

$I_{1} 1: r_{1}=r_{2}=r_{3}=r_{4}=2,3 \leq r_{5} \leq r_{6} \leq r_{7}$.

$I I_{1} 1: r_{1}=5, r_{2}=r_{3}=r_{4}=2,3 \leq r_{5} \leq r_{6}, I I_{1} 2: r_{1} \geq 7, r_{2}=2, r_{3}=r_{4}=$ $r_{5}=2, r_{6} \geq 3$.

$I I I_{1} 1: r_{1}=7, r_{2}=r_{3}=2,3 \leq r_{4} \leq r_{5}, I I I_{1} 2: r_{1}=8, r_{2}=r_{3}=r_{4}=$ $2, r_{5} \geq 3, I I I_{1} 3: r_{1} \geq 10, r_{2}=r_{3}=r_{4}=r_{5}=2$.

$I V_{1} 1: r_{1}=r_{2}=5, r_{3}=r_{4}=2, r_{5} \geq 3, I V_{1} 2: r_{1}=5, r_{2} \geq 7, r_{3}=r_{4}=$ $r_{5}=2$.

$V_{1} 1: r_{1}=9, r_{2}=2, r_{3} \geq 3, V_{1} 2: r_{1}=11, r_{2}=r_{3}=r_{4}=2$.

$V I_{1} 1: r_{1}=7, r_{2}=5, r_{3}=2, r_{4} \geq 3, V I_{1} 2: r_{1}=7, r_{2} \geq 7, r_{3}=r_{4}=2$, $V I_{1} 3: r_{1}=8, r_{2}=5, r_{3}=r_{4}=2$.

(5.3) Proof of the Theorem $\mathrm{C}$ for the cases of 5.2. Here we have $q_{1}=2, q_{2}=$ $3, q_{3} \geq 4$, and $D^{2}=3-\sum_{\alpha}\left(\left(i_{\alpha}\right)^{2} / r_{\alpha}\right)$. We can easily see $D^{2}>(1 / 12)$ for $\mathrm{II}_{1} 2$, $\mathrm{III}_{1} 3, \mathrm{IV}_{1} 2, \mathrm{VI}_{1} 2$. Hence $q_{1} q_{2} q_{3} D^{2}>2$ holds for these cases.

$\left(\mathrm{I}_{1} 1\right)$ Since $0<D^{2}=1-\left(1 / r_{5}\right)-\left(1 / r_{6}\right)-\left(1 / r_{7}\right)$, we have $r_{7} \geq 4$. Hence $b_{4}$ $=4-\left(4-r_{5}\right)_{+}-\left(4-r_{6}\right)_{+}-\left(4-r_{7}\right)_{+} \geq 2$, and $q_{3}=4$. Here $D^{2} \geq(1 / 12)$. Hence $q_{1} q_{2} q_{3} D^{2} \geq 2$. The equalities holds if and only if $r_{5}=r_{6}=3$, and $r_{7}=4$.

$\left(\mathrm{II}_{1} 1\right)$ We have $b_{4}=3-\left(4-r_{5}\right)_{+}-\left(4-r_{6}\right)_{+}$. If $b_{4} \geq 2$, then $r_{6} \geq 4$. We have $D^{2}=(7 / 10)-\left(1 / r_{5}\right)-\left(1 / r_{6}\right) \geq(7 / 60)>(1 / 12)$. Hence $q_{1} q_{2} q_{3} D^{2}>2$. Now assume $b_{4}=1$. Then $r_{1}=5, r_{2}=r_{3}=r_{4}=2, r_{5}=r_{6}=3$, and $D^{2}=1 / 30$. A direct computation shows $q_{3}=10$. Hence $q_{1} q_{2} q_{3} D^{2}=2$. 
$\left(\mathrm{III}_{1}\right)$ If $r_{5} \geq 4$, then $D^{2}=(5 / 7)-\left(1 / r_{4}\right)-\left(1 / r_{5}\right)>(1 / 12)$. Hence $q_{1} q_{2} q_{3} D^{2}>$ 2. If $r_{4}=r_{5}=3$, then $D^{2}=1 / 21$. A direct computation shows $q_{3}=7$. Hence $q_{1} q_{2} q_{3} D^{2}=2$.

$\left(\mathrm{III}_{1} 2\right)$ If $r_{5} \geq 4$, then $D^{2}=(3 / 8)-\left(1 / r_{5}\right)>(1 / 12)$. Hence $q_{1} q_{2} q_{3} D^{2}>2$. If $r_{5}$ $=3$, then $D^{2}=1 / 24$. A direct computation shows $q_{3}=8$. Hence $q_{1} q_{2} q_{3} D^{2}=2$. $\left(\mathrm{IV}_{1} 1\right)$ If $r_{5} \geq 4$, then $D^{2}=(2 / 5)-\left(1 / r_{5}\right) \geq(3 / 20)>(1 / 12)$. Hence $q_{1} q_{2} q_{3} D^{2}$ $>2$. If $r_{5}=3$, we have $D^{2}=1 / 15$. We can show $q_{3}=5$. Hence $q_{1} q_{2} q_{3} D^{2}=2$. $\left(\mathrm{V}_{1} 1\right)$ If $r_{4} \geq 4$, we have $D^{2}>(1 / 12)$. Hence $q_{1} q_{2} q_{3} D^{2}>2$. If $r_{3}=r_{4}=3$, then $D^{2}=1 / 18$. A direct computation shows $q_{3}=7$. Hence $q_{1} q_{2} q_{3} D^{2}>2$.

$\left(\mathrm{V}_{1} 2\right)$ We have $D^{2}=1 / 22$. A direct computation shows $q_{3}=8$. Hence $q_{1} q_{2} q_{3} D^{2}>2$.

$\left(\mathrm{VI}_{1} 1\right)$ If $r_{4} \geq 4$, then $D^{2}=(29 / 70)-\left(1 / r_{4}\right)>(1 / 12)$. Hence $q_{1} q_{2} q_{3} D^{2}>2$. If $r_{4}=3$, then $D^{2}=17 / 210$. We can show $q_{3}=5$. Hence $q_{1} q_{2} q_{3} D^{2}>2$.

$\left(\mathrm{VI}_{1} 3\right)$ Here we have $D^{2}=3 / 40$. A direct computation shows $q_{3}=5$. Hence $q_{1} q_{2} q_{3} D^{2}=2+(1 / 4)>2$.

Lemma 5.4. Assume $b_{3}=0$. Then, for the cases I-VI, one of the followings occurs.

$$
\begin{aligned}
& I_{0} 1: r_{1}=r_{2}=r_{3}=r_{4}=r_{5}=2,3 \leq r_{6} \leq r_{7} . \\
& I I_{0} 1: r_{1}=5, r_{2}=r_{3}=r_{4}=r_{5}=2,3 \leq r_{6}, I I_{0} 2: r_{1} \geq 9, r_{2}=2, r_{3}=r_{4}= \\
r_{5}= & r_{6}=2 . \\
& I I I_{0} 1: r_{1}=7, r_{2}=r_{3}=r_{4}=2,3 \leq r_{5}, I I I_{0} 2: r_{1}=8, r_{2}=r_{3}=r_{4}=r_{5}=
\end{aligned}
$$

2.

$$
\begin{aligned}
& I V_{0} 1: r_{1}=r_{2}=5, r_{3}=r_{4}=r_{5}=2 . \\
& V_{0} 1: r_{1}=9, r_{2}=r_{3}=2, r_{4} \geq 3 . \\
& V I_{0} 1: r_{1}=7, r_{2}=5, r_{3}=r_{4}=2 .
\end{aligned}
$$

(5.5) Proof of the Theorem $C$ for the cases of 5.4. Here we have $q_{1}=2, q_{3} \geq$ $q_{2} \geq 4$, and $D^{2}=3-\sum_{\alpha}\left(\left(i_{\alpha}\right)^{2} / r_{\alpha}\right)$. By this we can easily see $D^{2}<0$ for $\mathrm{III}_{0} 2, \mathrm{IV}_{0} 1, \mathrm{VI}_{0} 1$. Hence these cases do not happen.

$\left(\mathrm{I}_{0} 1\right)$ We have $b_{4}=2-\left(4-r_{6}\right)_{+}-\left(4-r_{7}\right)_{+}$.

If $b_{4}=2$, then $r_{6} \geq 4$ and $q_{2}=4$. Since $0<D^{2}=(1 / 2)-\left(1 / r_{6}\right)-\left(1 / r_{7}\right)$, we have $r_{7} \geq 5$. Here we can see $b_{5} \geq 1$ and $q_{3}=5$. Now $D^{2} \geq(1 / 20)$. Hence $q_{1} q_{2} q_{3} D^{2} \geq 2$. The equality holds if and only if $r_{6}=4, r_{7}=5$.

If $b_{4}=1$, then $r_{6}=3, r_{7} \geq 4$. Since $0<D^{2}=(1 / 6)-\left(1 / r_{7}\right)$, we have $r_{7} \geq 7$. Here we can see $b_{5}=0, b_{6}=2, b_{7}=1$ and $q_{2}=6, q_{3}=7$. Now $D^{2} \geq(1 / 42)$. Hence $q_{1} q_{2} q_{3} D^{2} \geq 2$. The equality holds if and only if $r_{7}=7$. $\left(\mathrm{II}_{0} 1\right)$ Since $0<D^{2}=(1 / 5)-\left(1 / r_{6}\right)$, we have $r_{6} \geq 6$. Here we can show $b_{4}=1, b_{5}=1, b_{6}=2$, and $q_{2}=5, q_{3}=6$. Now we have $D^{2} \geq(1 / 30)$. Hence $q_{1} q_{2} q_{3} D^{2} \geq 2$. The equality holds if and only if $r_{6}=6$. 
$\left(\mathrm{II}_{0} 2\right)$ Here we have $D^{2}=(1 / 2)-\left(4 / r_{1}\right) \geq(1 / 18)$. Further we can see $b_{4}=2$. Hence $q_{2}=4, q_{3} \geq 5$ and $q_{1} q_{2} q_{3} D^{2} \geq 40(1 / 18)>2$.

$\left(\mathrm{III}_{0} 1\right)$ Since $0<D^{2}=(3 / 14)-\left(1 / r_{5}\right)$, we have $r_{5} \geq 5$. Here we can show $b_{4}=b_{5}=1$, and $q_{2}=5, q_{3} \geq 6$.

If $r_{5} \geq 6$, then $D^{2} \geq(3 / 14)-(1 / 6)=(1 / 21)$. Hence $q_{1} q_{2} q_{3} D^{2} \geq 2 \cdot 5$. $6(1 / 21)>2$.

If $r_{5}=5$, we have $D^{2}=(1 / 70)$. A direct computation shows $q_{3}=14$. Hence $q_{1} q_{2} q_{3} D^{2}=2$.

$\left(\mathrm{V}_{0} 1\right)$ Since $0<D^{2}=(2 / 9)-\left(1 / r_{4}\right)$, we have $r_{4} \geq 5$. Here we can show $b_{4}=b_{5}=1$. Hence $q_{2}=5, q_{3} \geq 6$.

If $r_{4} \geq 6$, then $D^{2}>(1 / 30)$. Hence $q_{1} q_{2} q_{3} D^{2}>2$.

If $r_{4}=5$, then $D^{2}=(1 / 45)$. A direct computation shows $q_{3}=9$. Hence $q_{1} q_{2} q_{3} D^{2}=2$.

(5.6) Now one can show $q_{1} q_{2} q_{3} D^{2}>2$ for the cases VII-XV by similar arguments. These are not difficult. Hence we left the details to the readers and we omit them.

\section{$\S 6 . \quad$ In the Case with $b_{1}=b_{2}=1$}

In this section, we assume that $\underline{b}_{1}=1, b_{2}=1$. We have $q_{1}=1, q_{2} \geq 3$,

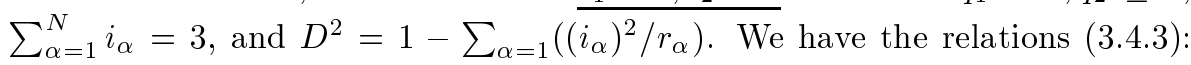
$b_{3}=2-\sum_{\alpha}\left(3 i_{\alpha}-r_{\alpha}\right)_{+}, b_{4}=4-\sum_{\alpha}\left(4 i_{\alpha}-r_{\alpha}\right)_{+}, b_{5}=7-\sum_{\alpha}\left(5 i_{\alpha}-r_{\alpha}\right)_{+}-$ $\sum_{\alpha}\left(5 i_{\alpha}-2 r_{\alpha}\right)_{+}, \ldots$. In the below we separate the arguments into cases depending on the type $\left(i_{1}, \ldots, i_{N}\right)$.

Case 6.1. $\quad N=3,\left(i_{1}, i_{2}, i_{3}\right)=(1,1,1)$, with $2 \leq r_{1} \leq r_{2} \leq r_{3}$.

We have $b_{3}=2-\sum_{\alpha=1}^{3}\left(3-r_{\alpha}\right)_{+}$.

(6.1.1) If $b_{3}=2$, then $r_{1} \geq 3$, and we have $q_{2}=3, q_{3} \geq 4$. We have $b_{4}=$ $4-\sum_{\alpha=1}^{3}\left(4-r_{\alpha}\right)_{+}$.

(6.1.1.1) If $b_{4} \geq 3$, then $r_{2} \geq 4, q_{3}=4$. We have $D^{2}=1-\left(1 / r_{1}\right)-\left(1 / r_{2}\right)-$ $\left(1 / r_{3}\right) \geq(1 / 6)$. Hence $q_{1} q_{2} q_{3} D^{2} \geq 2$. The equality holds if and only if $r_{1}=$ $3, r_{2}=r_{3}=4$.

(6.1.1.2) If $b_{4}=2$, then $r_{1}=r_{2}=3, r_{3} \geq 4, q_{3} \geq 5$.

(6.1.1.2.a) If $r_{3} \geq 6$, then $D^{2}=(1 / 3)-\left(1 / r_{3}\right) \geq(1 / 6)>(2 / 15)$. Hence $q_{1} q_{2} q_{3} D^{2} \geq 30 D^{2}>2$.

(6.1.1.2.b) If $r_{3}=5$, then $D^{2}=2 / 15$. A direct computation shows $q_{3}=5$. Hence $q_{1} q_{2} q_{3} D^{2}=2$.

(6.1.1.2.c) If $r_{3}=4$, then $D^{2}=1 / 12$. A direct computation shows $q_{3}=8$. Hence $q_{1} q_{2} q_{3} D^{2}=2$. 
(6.1.2) Now we assume $b_{3}=1$. Then $r_{1}=2, r_{2} \geq 3$ and we have $b_{4}=$ $2-\left(4-r_{2}\right)_{+}-\left(4-r_{3}\right)_{+}$.

(6.1.2.1) If $b_{4}=2$, then $r_{2} \geq 4, q_{2}=4$. We have $b_{5}=3-\left(5-r_{2}\right)_{+}-\left(5-r_{3}\right)_{+}$. (6.1.2.1.1) If $b_{5}=3$, then $r_{2} \geq 5$ and $q_{3}=5$. We have $D^{2}=(1 / 2)-\left(1 / r_{2}\right)-$ $\left(1 / r_{3}\right) \geq(1 / 10)$ hence $q_{1} q_{2} q_{3} D^{2} \geq 2$. Here the equality holds if and only if $r_{2}=r_{3}=5$.

(6.1.2.1.2) If $b_{5}=2$, then $r_{2}=4, r_{3} \geq 5$ and $q_{3} \geq 6$.

(6.1.2.1.2.a) If $r_{3} \geq 7$, then $D^{2} \geq(3 / 28)>(1 / 12)$. Hence $q_{1} q_{2} q_{3} D^{2}>2$.

(6.1.2.1.2.b) If $r_{3}=6$, then $D^{2}=1 / 12$. A direct computation shows $q_{3}=6$. Hence $q_{1} q_{2} q_{3} D^{2}=2$.

(6.1.2.1.2.c) If $r_{3}=5$, then $D^{2}=1 / 20$. A direct computation shows $q_{3}=10$. Hence $q_{1} q_{2} q_{3} D^{2}=2$.

(6.1.2.2) If $b_{4}=1$, then $r_{1}=2, r_{2}=3, r_{3} \geq 4$. Since $0<D^{2}=(1 / 6)-\left(1 / r_{3}\right)$, $r_{3} \geq 7$ holds. Here we can prove $b_{5}=1, b_{6}=2, b_{7}=2$, hence $q_{2}=6, q_{3} \geq 8$ hold.

(6.1.2.2.a) If $r_{3} \geq 9$, then $D^{2} \geq(1 / 18)>(1 / 24)$ and $q_{1} q_{2} q_{3} D^{2}>2$.

(6.1.2.2.b) If $r_{3}=8$, then $D^{2}=1 / 24$. A direct computation shows $q_{3}=8$, hence $q_{1} q_{2} q_{3} D^{2}=2$.

(6.1.2.2.c) If $r_{3}=7$, then $D^{2}=1 / 42$. A direct computation shows $q_{3}=14$, hence $q_{1} q_{2} q_{3} D^{2}=2$.

Case 6.2. $\quad N=2,\left(i_{1}, i_{2}\right)=(2,1)$, with $r_{1} \geq 5,\left(r_{1}, 2\right)=1,2 \leq r_{2}$.

We have $b_{3}=2-\left(6-r_{1}\right)_{+}-\left(3-r_{2}\right)_{+}$.

(6.2.1) If $b_{3}=2$, then $r_{1} \geq 7, r_{2} \geq 3$ and $q_{2}=3$. We have $b_{4}=4-\left(8-r_{1}\right)_{+}-$ $\left(4-r_{2}\right)_{+}$.

(6.2.1.1) If $b_{4} \geq 3$, then $q_{3}=4$ and either $r_{1} \geq 9, r_{2} \geq 3$ or $r_{1} \geq 7, r_{2} \geq 4$ hold. In both cases, we can easily show $D^{2}>(1 / 6)$, hence $q_{1} q_{2} q_{3} D^{2}>2$.

(6.2.1.2) If $b_{4}=2$, then $r_{1}=7, r_{2}=3$ and $D^{2}=2 / 21$. A direct computation shows $q_{3}=7$, hence $q_{1} q_{2} q_{3} D^{2}=2$.

(6.2.2) Now we assume $b_{3}=1$. Then we have two cases: (6.2.2.a) $r_{1}=5, r_{2} \geq 3$ or $(6.2 .2 . \mathrm{b}) r_{1} \geq 7, r_{2}=2$, occur.

(6.2.2.a) Since $0<D^{2}=(1 / 5)-\left(1 / r_{2}\right)$, we have $r_{2} \geq 6$. Here we can prove $b_{4}=1, b_{5}=2, b_{6}=2$, hence $q_{2}=5, q_{3} \geq 7$.

(6.2.2.a.a) If $r_{2} \geq 8$, then $D^{2} \geq(3 / 40)>(2 / 35)$ and $q_{1} q_{2} q_{3} D^{2}>2$.

(6.2.2.a.b) If $r_{2}=7$, then $D^{2}=2 / 35$. A direct computation shows $q_{3}=7$. Hence $q_{1} q_{2} q_{3} D^{2}=2$.

(6.2.2.a.c) If $r_{2}=6$, then $D^{2}=1 / 30$. A direct computation shows $q_{3}=12$. Hence $q_{1} q_{2} q_{3} D^{2}=2$.

(6.2.2.b) Since $0<D^{2}=(1 / 2)-\left(4 / r_{1}\right)$, we have $r_{1} \geq 9$. 
(6.2.2.b.a) If $r_{1} \geq 11$, then $D^{2} \geq(3 / 22)>(1 / 8)$. Hence $q_{1} q_{2} q_{3} D^{2} \geq 1 \cdot 4 \cdot 4 D^{2}>2$. (6.2.2.b.b) If $r_{1}=9$, then $D^{2}=1 / 18$. A direct computation shows $q_{2}=4, q_{3}=$ 9. Hence $q_{1} q_{2} q_{3} D^{2}=2$.

Case 6.3. $\quad N=1, i_{1}=3$, with $r_{1} \geq 7,\left(r_{1}, 3\right)=1$.

Since $0<D^{2}=1-\left(9 / r_{1}\right)$, we have $r_{1} \geq 10$. Here we have $b_{3}=2$, hence $q_{2}=3, q_{3} \geq 4$.

(6.3.a) If $r_{1} \geq 11$, then $D^{2} \geq(2 / 11)>(1 / 6)$ and $q_{1} q_{2} q_{3} D^{2} \geq 1 \cdot 3 \cdot 4 D^{2}>2$.

(6.3.b) If $r_{1}=10$, then $D^{2}=(1 / 10)$. A direct computation shows $q_{3}=7$. Hence $q_{1} q_{2} q_{3} D^{2}=2+(1 / 10)>2$.

This completes the proof of Theorem C.

\section{$\S 7 . \quad$ Simple K3 Singularities of Multiplicity Two}

(7.1) Now the proof of Theorem B is given as follows: Here we can show the equality $D^{2}=\lim _{\lambda \rightarrow 1}(1-\lambda)^{3} P(G, \lambda)(2.1)$. Since $e(m, A)=2$, we obtain the equality $2=\operatorname{deg} x_{1} \cdot \operatorname{deg} x_{2} \cdot \operatorname{deg} x_{3} \cdot \lim _{\lambda \rightarrow 1}(1-\lambda)^{3} P(G, \lambda)=e(m, A)$ by Theorem A (i). Therefore $2=e(m, A)=e\left(G_{+}, G\right)$ and there exists parameter system of $m \subset A$ whose initial form gives a parameter system of $G_{+} \subset G$. In particular, $G$ is a hypersurface and there is a system $y_{1}, y_{2}, y_{3}, y_{4}$ which generates $m \cdot A$ and initial form of them generates $G_{+}$. This completes the proof of Theorem B.

Now, for this special non-rational singularity, a conjecture of M. Reid (4.2) of [14] about the existence of such a good coordinates is proved in the following form.

Corollary 7.2. $\quad$ Let $(V, p)$ be a simple $K 3$ singularity of multiplicity two and $G$ the associated graded ring of the canonical filtration. Then in the coordinate which induced from the homogeneous minimal generator of $G_{+}$, there is a 3-dimensional compact face $\Gamma_{0}$ of the Newton boundary of the defining equation of $V$ where $(1,1,1,1) \in \mathbf{R}^{4}$ is contained in the relative interior of $\Gamma_{0}$.

Proof. Let $V=\left\{\left(y_{1}, y_{2}, y_{3}, y_{4}\right) \in \mathbf{C}^{4} \mid f\left(y_{1}, y_{2}, y_{3}, y_{4}\right)=0\right\}$ be the representation of $(V, p)$ by the new coordinate $\left(y_{1}, y_{2}, y_{3}, y_{4}\right)$ in the arguments of (7.1). Let $f=\sum_{k \geq 0} f_{k}$ be the weighted Taylor expansion with respect to the $w t\left(y_{i}\right)=q_{i}$, for $i=1,2,3,4$, and $f_{h}$ the initial form. The proof of Theorem B implies that $G=R(E, D) \cong \mathbf{C}\left[y_{1}, y_{2}, y_{3}, y_{4}\right] / f_{h}$. Therefore $f_{h} \in \mathbf{C}\left[y_{1}, y_{2}, y_{3}, y_{4}\right]$ is a quasi-homogeneous polynomial with $h=\sum_{1 \leq i \leq 4} q_{i}$ 
and $\left\{f_{h}=0\right\}-\{o\}$ has only rational singularities (see $[19,(1.5)]$ ). By Theorem 5.6 of [19], the Newton boundary $\Gamma\left(f_{h}\right) \subset \mathbf{R}^{4}$ is 3-dimensional and $(1,1,1,1)$ is contained in the relative interior of $\Gamma\left(f_{h}\right)$. Now the assertion on the Newton boundary of $f$ follows as $\Gamma_{0}=\Gamma\left(f_{h}\right)$.

(7.3) A new proof of classification of the lists of weights for simple K3 double points. As noted in Introduction, there are two basic works [2] and [23] on classification of hypersurface simple K3 singularities. In both studies, the list of famous 95 weights is created from the studies on the weight systems on the coordinates. In [2], Fletcher gives the list as that of certain quasi-smooth weight hypersurfaces which give normal K3 surfaces, and in [23], Yonemura gives the same list from the points of classification of special convex polytopes which corresponds to the initial form of non-degenerate functions. Now, for the cases of multiplicity two, we can give the list as a corollary of the proof of Theorem C. We obtain the list of 48 cases with the datum $D^{2}, q_{1}, q_{2}, q_{3}$ with the baskets of singularities where the relation $q_{1} q_{2} q_{3} D^{2}=2$ holds. Set $q_{4}$ as $q_{4}=q_{1}+q_{2}+q_{3}$ and set $h$ as $h=2 q_{4}$. Then the set of these $\left(q_{1}, q_{2}, q_{3}, q_{4} ; h\right)$ gives the list for weights of simple K3 singularities of multiplicity two.

The data for the cases with $q_{1} q_{2} q_{3} D^{2}=2$

\begin{tabular}{|c|c|c|l|l|}
\hline reference & $D^{2}$ & $q_{1}, q_{2}, q_{3}$ & $\left(r_{\alpha}, i_{\alpha}\right): c l(D)=i_{\alpha} \in \mathbf{Z} / r_{\alpha} \mathbf{Z}$ & Example of $E$ \\
\hline$(3.5)$ & $\frac{1}{6}$ & $2,2,3$ & $(2,1) \times 7,(3,1)$ & $X_{14} \subset P(2.2 .3 .7)$ \\
$(3.6)$ & 2 & $1,1,1$ & $D$ is Cartier & $X_{6} \subset P(1.1 .1 .3)$ \\
$(3.7)$ & 1 & $1,1,2$ & $(2,1) \times 2$ & $X_{8} \subset P(1.1 .2 .4)$ \\
$(3.7)$ & $\frac{2}{3}$ & $1,1,3$ & $(3,1)$ & $X_{10} \subset P(1.1 .3 .5)$ \\
$(3.7)$ & $\frac{1}{2}$ & $1,1,4$ & $(2,1)$ & $X_{12} \subset P(1.1 .4 .6)$ \\
$(3.9)$ & $\frac{1}{2}$ & $1,2,2$ & $(2,1) \times 5$ & $X_{10} \subset P(1.2 .2 .5)$ \\
$(3.10 .1 .1)$ & $\frac{1}{3}$ & $1,2,3$ & $(2,1) \times 2,(3,1) \times 2$ & $X_{12} \subset P(1.2 .3 .6)$ \\
$(3.10 .1 .2 .1)$ & $\frac{1}{4}$ & $1,2,4$ & $(2,1) \times 3,(4,1)$ & $X_{14} \subset P(1.2 .4 .7)$ \\
$(3.10 .1 .2 .2)$ & $\frac{1}{6}$ & $1,2,6$ & $(2,1) \times 3,(3,1)$ & $X_{18} \subset P(1.2 .6 .9)$ \\
$(3.10 .2 .2)$ & $\frac{1}{5}$ & $1,2,5$ & $(5,2),(2,1) \times 2$ & $X_{16} \subset P(1.2 .5 .8)$ \\
$\left(4.7 . \mathrm{I}_{0,1} 1\right)$ & $\frac{1}{60}$ & $4,5,6$ & $(2,1) \times 2,(3,1),(4,1)$, & $X_{30} \subset P(4.5 .6 .15)$ \\
$\left(4.3 . \mathrm{II}_{1,1} 1\right)$ & $\frac{1}{30}$ & $3,4,5$ & $(5,1) \times 2$ & \\
$\left(4.3 . \mathrm{II}_{1,1} 2\right)$ & $\frac{1}{84}$ & $3,4,14$ & $(7,2),(3,1) \times 2,(4,1),(3,1) \times 2$, & $X_{24} \subset P(3.4 .5 .12)$ \\
& & $(4,1)$ & $X_{42} \subset P(3.4 .14 .21)$ \\
$\left(4.7 . \mathrm{II}_{0,1} 2\right)$ & $\frac{1}{84}$ & $4,6,7$ & $(7,2),(2,1) \times 2,(4,1)$, & $X_{34} \subset P(4.6 .7 .17)$ \\
$\left(4.5 . \mathrm{III}_{0,1} 1\right)$ & $\frac{1}{120}$ & $3,5,16$ & $(8,3),(3,1) \times 2,(5,1)$ & $X_{48} \subset P(3.5 .16 .24)$ \\
$\left(4.3 . \mathrm{III}_{1,1} 2\right)$ & $\frac{1}{60}$ & $3,4,10$ & $(10,3),(2,1),(3,1),(4,1)$ & $X_{34} \subset P(3.4 .10 .17)$ \\
$\left(4.3 . \mathrm{III}_{1,1} 3\right)$ & $\frac{1}{66}$ & $3,4,11$ & $(11,3),(2,1),(3,1) \times 2$ & $X_{36} \subset P(3.4 .11 .18)$ \\
\hline
\end{tabular}

(continued) 
(continued)

\begin{tabular}{|c|c|c|c|c|}
\hline reference & $D^{2}$ & $q_{1}, q_{2}, q_{3}$ & $\left(r_{\alpha}, i_{\alpha}\right): c l(D)=i_{\alpha} \in \mathbf{Z} / r_{\alpha} \mathbf{Z}$ & Example of $E$ \\
\hline$\left(4.7 . \mathrm{III}_{0,1} 1\right)$ & $\frac{1}{70}$ & $4,5,7$ & $(7,3),(4,1) \times 2,(5,1)$ & $X_{32} \subset P(4.5 .7 .16)$ \\
\hline$\left(4.9 . \mathrm{III}_{0,0} 2\right)$ & $\frac{1}{120}$ & $5,6,8$ & $(8,3),(2,1),(5,1),(6,1)$ & $X_{38} \subset P(5.6 .8 .19)$ \\
\hline$\left(4.3 . \mathrm{IV}_{1,1} 3\right)$ & $\frac{1}{42}$ & $3,4,7$ & $(7,2) \times 2,(2,1),(3,1)$ & $X_{28} \subset P(3.4 .7 .14)$ \\
\hline$\left(4.9 . \mathrm{IV}_{0,0} 1\right)$ & $\frac{1}{140}$ & $5,7,8$ & $(5,2) \times 2,(4,1),(7,1)$ & $X_{40} \subset P(5.7 .8 .20)$ \\
\hline$\left(4.7 \cdot \mathrm{IV}_{0,1} 1\right)$ & $\frac{1}{180}$ & $4,5,18$ & $(5,2),(9,2),(2,1),(4,1)$ & $X_{54} \subset P(4.5 .18 .27)$ \\
\hline$\left(4.9 . \mathrm{IV}_{0,0} 2\right)$ & $\frac{1}{280}$ & $7,8,10$ & $(5,2),(7,2),(2,1),(8,1)$ & $X_{50} \subset P(7.8 .10 .25)$ \\
\hline$\left(4.9 . \mathrm{IV}_{0,0} 3\right)$ & $\frac{1}{330}$ & $5,6,22$ & $(5,2),(11,2),(2,1),(3,1)$ & $X_{66} \subset P(5.6 .22 .33)$ \\
\hline$\left(4.5 \cdot V_{1,1} 1\right)$ & $\frac{2}{165}$ & $3,5,11$ & $(11,4),(3,1),(5,1)$ & $X_{38} \subset P(3.5 .11 .19)$ \\
\hline$\left(4.7 . \mathrm{VI}_{0,1} 2\right)$ & $\frac{1}{130}$ & $4,5,13$ & $(13,3),(5,2),(2,1)$ & $X_{44} \subset P(4.5 .13 .22)$ \\
\hline$\left(5.3 . \mathrm{I}_{1} 1\right)$ & $\frac{1}{12}$ & $2,3,4$ & $(2,1) \times 4,(3,1) \times 2,(4,1)$ & $X_{18} \subset P(2.3 .4 .9)$ \\
\hline$\left(5.5 . \mathrm{I}_{0} 1\right)$ & $\frac{1}{20}$ & $2,4,5$ & $(2,1) \times 5,(4,1),(5,1)$ & $X_{22} \subset P(2.4 .5 .11)$ \\
\hline$\left(5.5 . \mathrm{I}_{0} 1\right)$ & $\frac{1}{42}$ & $2,6,7$ & $(2,1) \times 5,(3,1),(7,1)$ & $X_{30} \subset P(2.6 .7 .15)$ \\
\hline$\left(5.3 . \mathrm{II}_{1} 1\right)$ & $\frac{1}{30}$ & $2,3,10$ & $(5,2),(2,1) \times 3,(3,1) \times 2$ & $X_{30} \subset P(2.3 .10 .15)$ \\
\hline$\left(5.5 . \mathrm{II}_{0} 1\right)$ & $\frac{1}{30}$ & $2,5,6$ & $(5,2),(2,1) \times 4,(6,1)$ & $X_{26} \subset P(2.5 .6 .13)$ \\
\hline$\left(5.3 . \mathrm{III}_{1} 1\right)$ & $\frac{1}{21}$ & $2,3,7$ & $(7,3),(2,1) \times 2,(3,1) \times 2$ & $X_{24} \subset P(2.3 .7 .12)$ \\
\hline$\left(5.3 . \mathrm{III}_{1} 2\right)$ & $\frac{1}{24}$ & $2,3,8$ & $(8,3),(2,1) \times 3,(3,1)$ & $X_{26} \subset P(2.3 .8 .13)$ \\
\hline$\left(5.5 . \mathrm{III}_{0} 1\right)$ & $\frac{1}{70}$ & $2,5,14$ & $(7,3),(2,1) \times 3,(5,1)$ & $X_{42} \subset P(2.5 .14 .21)$ \\
\hline$\left(5.3 . \mathrm{IV}_{1} 1\right)$ & $\frac{1}{15}$ & $2,3,5$ & $(5,2) \times 2,(2,1) \times 2,(3,1)$ & $X_{20} \subset P(2.3 .5 .10)$ \\
\hline$\left(5.5 . \mathrm{V}_{0} 1\right)$ & $\frac{1}{45}$ & $2,5,9$ & $(9,4),(2,1) \times 2,(5,1)$ & $X_{32} \subset P(2.5 .9 .16)$ \\
\hline$(6.1 .1 .1)$ & $\frac{1}{6}$ & $1,3,4$ & $(3,1),(4,1) \times 2$ & $X_{16} \subset P(1.3 .4 .8)$ \\
\hline$(6.1 .1 .2 . b)$ & $\frac{2}{15}$ & $1,3,5$ & $(3,1) \times 2,(5,1)$ & $X_{18} \subset P(1.3 .5 .9)$ \\
\hline (6.1.1.2.c) & $\frac{1}{12}$ & $1,3,8$ & $(3,1) \times 2,(4,1)$ & $X_{24} \subset P(1.3 .8 .12)$ \\
\hline$(6.1 .2 .1 .1)$ & $\frac{1}{10}$ & $1,4,5$ & $(2,1),(5,1) \times 2$ & $X_{20} \subset P(1.4 .5 .10)$ \\
\hline$(6.1 .2 .1 .2 . \mathrm{b})$ & $\frac{1}{12}$ & $1,4,6$ & $(2,1),(4,1),(6,1)$ & $X_{22} \subset P(1.4 .6 .11)$ \\
\hline$(6.1 .2 .1 .2 . c)$ & $\frac{1}{20}$ & $1,4,10$ & $(2,1),(4,1),(5,1)$ & $X_{30} \subset P(1.4 .10 .15)$ \\
\hline$(6.1 .2 .2 . \mathrm{b})$ & $\frac{1}{24}$ & $1,6,8$ & $(2,1),(3,1),(8,1)$ & $X_{30} \subset P(1.6 .8 .15)$ \\
\hline$(6.1 .2 .2 . \mathrm{c})$ & $\frac{1}{42}$ & $1,6,14$ & $(2,1),(3,1),(7,1)$ & $X_{42} \subset P(1.6 .14 .21)$ \\
\hline$(6.2 .1 .2)$ & $\frac{2}{21}$ & $1,3,7$ & $(7,2),(3,1)$ & $X_{22} \subset P(1.3 .7 .11)$ \\
\hline$(6.2 .2 . \mathrm{a} . \mathrm{b})$ & $\frac{2}{35}$ & $1,5,7$ & $(5,2),(7,1)$ & $X_{26} \subset P(1.5 .7 .13)$ \\
\hline (6.2.2.a.c) & $\frac{1}{30}$ & $1,5,12$ & $(5,2),(6,1)$ & $X_{36} \subset P(1.5 .12 .18)$ \\
\hline$(6.2 .2 . \mathrm{b} . \mathrm{b})$ & $\frac{1}{18}$ & $1,4,9$ & $(9,2),(2,1)$ & $X_{28} \subset P(1.4 .9 .14)$ \\
\hline
\end{tabular}

Here $X_{h} \subset P\left(q_{1}, q_{2}, q_{3}, q_{4}\right)$ denotes a weight hypersurface defined by a quasihomegeneous polynomial $f_{h} \in \mathbf{C}\left[y_{1}, y_{2}, y_{3}, y_{4}\right]$ of type $\left(q_{1}, q_{2}, q_{3}, q_{4} ; h\right)$ as in $(7.2)$.

Here one can find many examples of simple K3 singularity for each weight in the lists of Fletcher [2]-Yonemura [23]. By the existence of such weighted hypersurfaces, we can show the following where the $\operatorname{ring} G$ is not necessarily a hypersurface, but the Poincare series for $G$ is very simple.

Corollary 7.4. Let $G=R(E, D)$ be a normal graded ring with $E$ a normal K3 surface and $D$ an integral Weil divisor. Suppose $q_{1} q_{2} q_{3} D^{2}=2$ 
holds. Then the Poincare series $P(G, \lambda)$ is given by the following way:

$$
P(G, \lambda)=\frac{1-\lambda^{2\left(q_{1}+q_{2}+q_{3}\right)}}{\left(1-\lambda^{q_{1}}\right)\left(1-\lambda^{q_{2}}\right)\left(1-\lambda^{q_{3}}\right)\left(1-\lambda^{q_{1}+q_{2}+q_{3}}\right)} .
$$

Proof. By the list (7.3), the basket of singularities is determined by $q_{1}, q_{2}, q_{3}$ and $D^{2}$. Hence $P(G, \lambda)$ is determined. Let $q_{4}=q_{1}+q_{2}+q_{3}$, and $h=2 q_{4}$. Then for each data, we can find a quasi-homogeneous polynomial of type $\left(q_{1}, q_{2}, q_{3}, q_{4} ; h\right)$ with isolated singularity by Fletcher [2] and Yonemura [23]. Hence, for each case, the Poincare series $P(G, \lambda)$ is given as in the assertion by $(2.2)$.

The following is a corollary of S. Ishii's theory and our main theorem. The following theorem say "the weight type of the simple K3 double point stays the same under arbitrary one-parameter $(F G)$-deformation".

Theorem 7.5. Let $\pi: \mathcal{V} \rightarrow T \subset \mathbf{C}$ be a one-parameter family of simple K3 double points such that the resolution $\psi: \tilde{\mathcal{V}} \rightarrow \mathcal{V}$ has the relative canonical model, i.e., $\pi$ is an $(F G)$-deformation after Ishii [8]. Assume that non-rational singularity of $V_{t}$ appears along a section $P: T \rightarrow \mathcal{V}$. Let $0 \in T$, then in a neighborhood of $P(0)$, there is a good coordinate $\left(x_{1}, x_{2}, x_{3}, x_{4}, t\right)$ such that $\pi$ is written as $\mathcal{V}=\left\{f\left(x_{1}, x_{2}, x_{3}, x_{4}, t\right)=0\right\} \ni\left(x_{1}, x_{2}, x_{3}, x_{4}, t\right) \rightarrow t \in T$ and the initial compact face $\Gamma_{0}(t)$ of $\Gamma\left(f_{t}\right)$ with respect to $\left(x_{1}, x_{2}, x_{3}, x_{4}\right)$ such that $\Gamma_{0}(t) \ni(1,1,1,1)$ and belongs to the hypersurface which is independent of $t \in T$.

Proof. In [8, Corollary 1.11, Theorem 2], S. Ishii showed that the $\pi$ admits the simultaneous canonical model and $\gamma_{m}\left(\mathcal{V}_{t}, P(t)\right)$ is constant. Let $F:(\mathcal{Y}, \mathcal{E}) \rightarrow(\mathcal{V}, P(T))$ be the simultaneous canonical model. Ishii's arguments show that $\mathcal{E} \rightarrow T$ is a family of normal K3 surfaces and $\mathcal{F}^{k}=F_{*}\left(O_{\mathcal{Y}}(-k \mathcal{E})\right) \subset$ $O_{\mathcal{V}}$ for $k \geq 1$ define the canonical filtration for each $t \in T$, and $\mathcal{F}^{k} / \mathcal{F}^{k+1}$ are locally free $O_{T}$-modules. Hence there are $x_{i} \in \mathcal{F}^{q_{i}}-\mathcal{F}^{q_{i}+1}, i=1,2,3,4 \mathrm{such}$ that $x_{1}, x_{2}, x_{3}$ defines a homogeneous parameter of $g r_{\mathcal{F}} O_{\mathcal{V}} \otimes \mathbf{C}=R\left(\mathcal{E}_{0}, D\right)$ with $q_{1} q_{2} q_{3} D^{2}=2$ and $\left(x_{1}, x_{2}, x_{3}, x_{4}\right)$ gives a homogeneous generator of the maximal ideal of $g r_{\mathcal{F}} O_{\mathcal{V}} \otimes \mathbf{C}$. This properties are preserved for $t \in T$ in a neighborhood of 0 . Such a parameter of $g r_{\mathcal{F}} O_{\mathcal{V}} \otimes \mathbf{C}_{t}$ determines the good coordinates in the sense of (7.2). Hence in the coordinate $\left(x_{1}, x_{2}, x_{3}, x_{4}, t\right)$, we obtain the desired properties.

Remark 7.6. (i) For deformations of simple K3 singularities of multiplicity three, the situation is not so simple as in (7.5), even we assume $\gamma_{m}$ 
is constant [9]. (ii) For some special simple K3 singularities, in some case for more general deformations, "the constant-ness of weight of simple K3 singularity" are studied by S. Ishii [8, Example 2.7], Y. Kaneko and M. Furuya [3], independently. In their studies, they assumed non-degenerate conditions of Newton boundary or quasi-homogeneous isolated-ness for the singularities.

\section{Acknowledgement}

The author heartily thanks to Prof. Kei-ichi Watanabe for many discussion on this subject and warm encouragements in these 15 years from the beginning of [20]. The author also thanks the members of Seminars at University of Tsukuba, Waseda Tuesday Seminar, and Algebraic Geometry Seminar at Kanazawa University.

\section{References}

[1] Demazure, M., Anneaux gradués normaux; in Seminarire Demazure -Giraud -Teissier, 1979. Ecole Polytechnique In: Lê Dũng Tráng (ed.), Introduction a la théorie des singularités II; Méthodes algébriques et géométriques, (Travaux En Cours. 37, pp.35-68) Paris: Hermann 1988.

[2] Fletcher, A. R., Plurigenera of 3-folds and weighted hypersurfaces, Thesis submitted to the University of Warwick for the degree of Ph.D., 1988.

[3] Furuya, M., On $\delta_{m}$-constant locus of versal deformations of non-degenerate hypersurface simple K3 singularities, Preprint, a version is alg-geom/9702018.

[4] Giraud, J., Improvement of Grauert-Riemenschneider's Theorem for a normal surface, Ann. Inst. Fourier, Grenoble, 324 (1982), 13-23.

[5] Herrmann, M., Ikeda, S. and Orbanz, U., Equimultiplicity and blowing-up, Springer Verlag, 1988, p. 629.

[6] Ishii, S., On isolated Gorenstein singularities, Math. Ann., 270 (1985), 541-554.

[7] — - Isolated Q-Gorenstein singularities of dimension three, In: T. Suwa, Ph. Wagreich, (eds.), Complex Analytic Singularities: Japan-US Seminar 1984, Tsukuba Kyoto. (Adv. Stud. Pure Math., 8, pp. 165-198), Tokyo Amsterdam: Kinokuniya-NorthHolland, 1986.

[8] — - Simultaneous canonical models of deformations of isolated singularities, In: A. Fujiki, K. Kato, T. Katsura, Y. Kawamata and Y. Miyaoka, (eds.), Algebraic geometry and analytic geometry: Proceedings of a conference held in Tokyo, Japan august 13-17, 1990. ICM-90 Satellite conference proceedings, Springer-Verlag Tokyo, 1991.

[9] Ishii, S. and Tomari, M., Hypersurface non-rational singularities which look canonical from their Newton boundaries, Math. Z., 237 (2001), 125-147.

[10] Ishii, S. and Watanabe, Kimio, A geometric characterization of simple K3-singularities, Tohoku Math. J., 44 (1992), 19-24.

[11] Kawamata, Y., Matsuda, K. and Matsuki, K., Introduction to the minimal model problem, In: T. Oda, (ed.), Algebraic Geometry, Sendai, 1985. (Adv. Stud. Pure Math. 10, pp. 283-360), Tokyo Amsterdam: Kinokuniya-North-Holland, 1987.

[12] Matsumura, H., Commutative ring theory, Cambridge Stud. Adv. Math., 8 (1980 published in Japan, translation by M. Reid and published in 1986).

[13] Ramanujan, C. P., On a geometric interpretation of multiplicity, Invent. Math., 22 (1973), 63-67. 
[14] Reid, M., Canonical 3-folds, In: A. Beauville (ed.), Journées de géométrie algébrique d'Angers 1979, Algebraic geometry. pp. 273-310, Sijthhoff and Noordhoff (1980).

[15] — Y Young person's guide to canonical singularities, In: Bloch, S. J. (ed.), Algebraic Geometry, Bowdoin 1985, Part I (Proc. Sympos. Pure Math., 46, 354-414), Amer. Math. Soc. (1987).

[16] Rees, D., a-transforms of local rings and a theorem on multiplicities, Proc. Cambrige Philos. Soc., 57 (1961), 8-17.

[17] Saito, K., Quasihomogene isolierte Singularitäten von Hyperflächen, Invent. Math., 14 (1971), 123-142.

[18] - Einfach elliptische Singularitäten, Invent. Math., 23 (1974), 289-325.

[19] Tomari, M., The canonical filtration of higher dimensional purely elliptic singularity of a special type, Invent. Math., 104 (1991), 497-520.

[20] Tomari, M. and Watanabe, Kei-ichi, Filtered rings, filtered blowing-ups and normal two-dimensional singularities with "star-shaped" resolution, Publ. RIMS, Kyoto Univ., 25 (1989), 681-740.

[21] Watanabe, Kei-ichi, Some remarks concerning Demazure's construction of normal graded rings, Nagoya Math. J., 83 (1981), 203-211.

[22] Watanabe, Kimio, On plurigenera of normal isolated singularities I, II, I. Math. Ann., 250 (1980), 65-94, II. In: T. Suwa, Ph. Wagreich, (eds.), Complex Analytic Singularities: Japan-US Seminar 1984, Tsukuba Kyoto (Adv. Stud. Pure Math. 8, pp. 671-685) Tokyo Amsterdam: Kinokuniya-North-Holland, 1986.

[23] Yonemura, T., On hypersurface simple K3-singularities, Tohoku Math. J., 42 (1990), 351-380. 